\title{
Utilisation De L'image Multispectrale Pour L'exploration Et La Recherche Des Ressources Minérales : État Des Connaissances Et Proposition D'un Modèle De Traitement
}

\author{
Abdessamad El Atillah \\ Zine El Abidine El Morjani \\ Mustapha Souhassou \\ EGERNE - Faculté Polydisciplinaire de Taroudant
}

Doi: 10.19044/esj.2018.v14n24p350 URL:http://dx.doi.org/10.19044/esj.2018.v14n24p350

\begin{abstract}
Multiband space remote sensing is an indirect tool for prospecting the Earth's surface. It is very powerful especially in its applications related to the field of geology including geological mapping, mining and oil exploration. It can also significantly reduce the cost of exploration, reach inaccessible areas, guide mining research to favorable regions and reach a large surface. In this article, we highlight in details the state of knowledge in this field of research by citing the different methods and approaches carried out by several specialists who generally define the use of remote sensing for lithostructural and mineralogical mapping and particularly for the exploration and research of mineral substances. We also create methods derived from the aforementioned methods of treatment by means of a logical analogy between the different bands of several satellites of observation of the terrestrial globe, particularly between : Landsat 7 ETM +; Landsat 8 OLI / TIRS; Aster and Sentinel 2A. At the end, we synthesize these results by proposing a multispectral image-processing model that can be applied directly. This model starts with the calculation of Optimum Index Factor (OIF), which allows us to detect only the most important colored composites; and the reports of the bands, rations, the principal component analysis, ACI and the classification that allow the realization of a lithological and mineralogical mapping as well as maps of lineaments by means of directional filters. The validity of the models is tested by comparison with field data and geological maps of the studied site.
\end{abstract}

Keywords: Spatial remote sensing, multispectral image, exploration, research and mineral resources. 


\section{Résumé}

La télédétection spatiale multibande est un outil indirect de prospection de la surface terrestre. Elle est très performante surtout dans des applications liées au domaine de la géologie notamment la cartographie géologique, l'exploration minière et pétrolière. Elle permet ainsi de réduire considérablement le coût d'exploration, en orientant la recherche minière vers les régions favorables d'une grande surface, qui sont parfois inaccessibles. Dans cet article, on va détailler l'état des connaissances dans ce domaine de recherche en citant les différentes méthodes et approches réalisées par plusieurs spécialistes qui définissent de près l'utilisation de la télédétection pour la cartographie lithostructurale et minéralogique, en général, et l'exploration et la recherche des substances minérales, en particulier. On créera, par la suite des méthodes dérivées à partir des méthodes de traitement précitées par le biais d'une analogie logique entre les différentes bandes de plusieurs satellites d'observation du globe terrestre notamment entre : Landsat 7 ETM+ ; Landsat 8 OLI /TIRS ; Aster et Sentinel 2A. En fin, on synthétise ces résultats à travers la proposition d'un modèle de traitement des images multispectrales qui peut être appliqué directement. Le calcul d'Optimum Index Factor (OIF) permettra de choisir les composites colorées les plus importantes; les rapports des bandes, rations, l'analyse en composante principale ACP, ACI et la classification ont permis la réalisation d'une cartographie lithologique et minéralogique ainsi que des cartes de linéaments par le biais des filtres directionnels. La validité des modèles est testée par comparaison avec les données de terrain et les cartes géologiques du site étudié.

Mots clés : Télédétection spatiale, image multispectrale, exploration, recherche et ressources minérales.

\section{Introduction}

La contribution de la géomatique associée à d'autres approches intégrées ( la géophysique et la géochimie) devrait être étudiées profondément pour adopter et développer une approche multidisciplinaire pour la prospection des gisements métallifères. C'est dans cette perspective que la télédétection et ses applications jouent un rôle très important en prospection géologique. La figure 1 résume les différentes applications de la télédétection en géologie. 


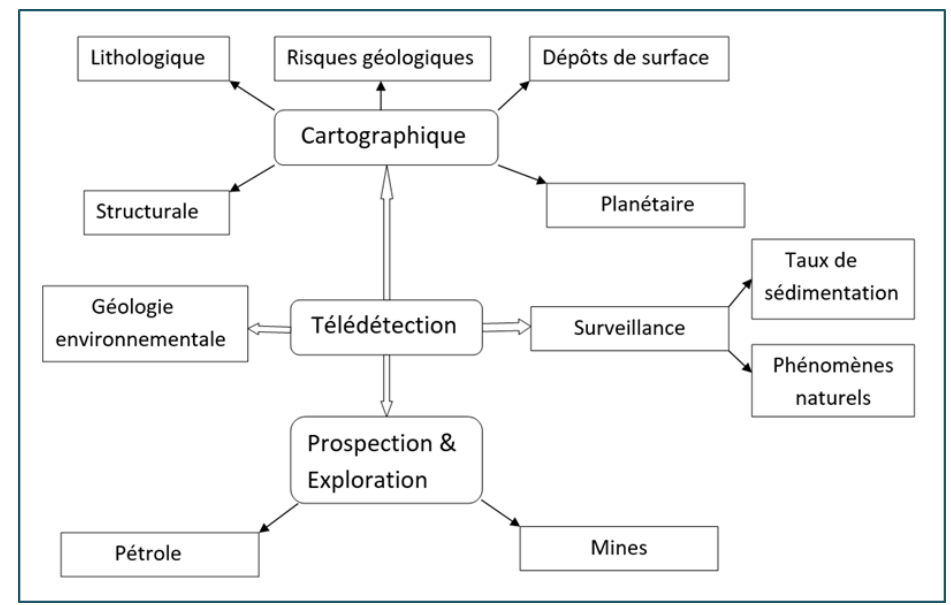

Figure 1 : Les principaux domaines d'applications de la télédétection en géologie (Taibi 2009)

La télédétection est un outil indirect de la prospection locale. Elle est très performante surtout dans ses applications en géologie: cartographie géologique, exploration minière et pétrolière et la prospection d'eau. En géologie minière, le traitement des images satellitaires permet l'individualisation des différents minéraux localisés à la surface.

La télédétection présente un grand intérêt à la réduction considérable du coût d'exploration, d'accéder aux zones à accès difficile, et d'orienter la prospection minière dans les régions potentiellement favorables à la mise en place de la minéralisation. L'avantage aussi que présente la télédétection est qu'elle permet l'investigation de l'ensemble des données par la fusion des données optiques et radar. La figure 2 présente les différents aspects de cette discipline.

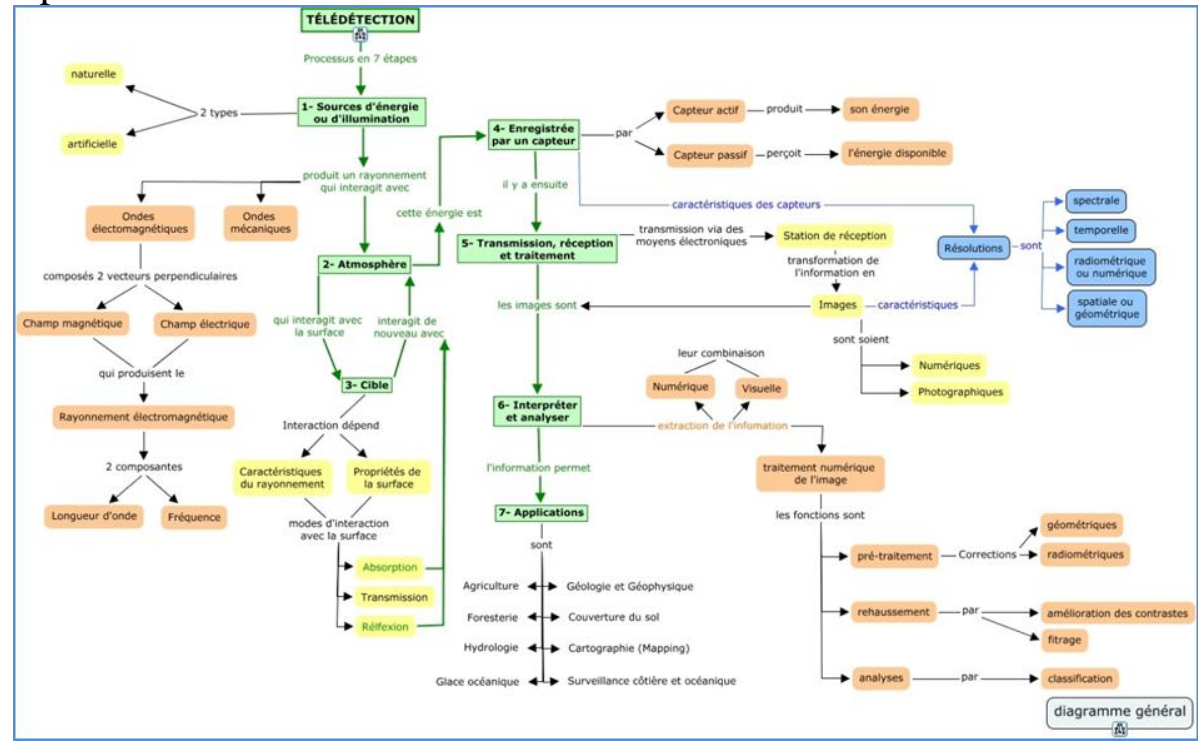

Figure 2 : Les différents aspects de la télédétection (IHMC Public Cmaps). 
Grace à la géomatique et ses outils, divers traitements peuvent être réalisés, allant des traitements les plus simples jusqu'à l'analyse spatiale basée sur les théories des statistiques multidimensionnelles (ONHYM ; 2018). Elle est généralement basée sur :

- Le traitement des images satellitaires, (Landsat, Sentinelle, Spot, Aster, Hyperion, etc), et aériennes, par une multitude de logiciels (exp : Erdas Imagine, Imagine OrthoBase, Imagine stereoAnalyst, Imagine virtual Gis, Rsi Envi).

- Spectroradiométrie par ASD (Analyse spectral Devices), et traitement des images hyperspectrales par les logiciels : Erdas Imagine, Rsi Envi, SII SpecMin et SII Specwin.

- Orthophogrammétrie par les logiciels : Erdas Imagine, Imagine OrthoBase, Rsi Envi, ESRI Arcgis, Esri 3D Analyst.

Dans le domaine de la géologie, Landsat et Aster restent les satellites d'observation de de Terre les plus utilisés.

Ce travail s'inscrit dans le cadre de l'utilisation de la géomatique visant à orienter les prospecteurs vers des zones susceptibles de contenir des concentrations de minerais. Il mettra à la disposition des opérateurs miniers et des juniors d'exploration un outil d'aide à la décision qui aura l'avantage d'être économique, rapide mais surtout applicable dans les zones reculées et inaccessibles.

C'est dans ce sens que nous proposons une démarche intégrée de traitement numérique des données spatiales pour répondre à notre problématique tout en mettant en œuvre des outils modernes d'analyse et de modélisation d'où la nécessité de cette recherche bibliographique pour l'élaboration d'un modèle de traitement. Ce travail nous permet d'encadrer cette thématique dans son environnement scientifique.

\section{Traitement des images du Landsat :}

Le programme Landsat a montré une bonne évolution en matière de capteurs (RBV, MSS, TM, ETM+, OLI, Thermal Infrared (TIRS)).Le tableau 1, ci-après, résume la particularité du Landsat TM et ses capteurs pour l'exploration et la recherche des substances minérales.

Tableau 1: Les différentes applications des images Landsat Sensor (Hamzaoui, 2005).

\begin{tabular}{|c|c|l|l|}
\hline Bande & $\begin{array}{c}\text { Longueur d'onde } \\
(\mu \mathrm{m})\end{array}$ & Résolution & \multicolumn{1}{c|}{ Application } \\
\hline TM 1 & $0.45-0.52($ Bleu $)$ & $30 \mathrm{~m}$ & $\begin{array}{l}\text { Discrimination du sol et de la végétation ; cartographie } \\
\text { côtière et bathymétrique ; identification des caractéristiques } \\
\text { urbaines et culturelles. }\end{array}$ \\
\hline TM 2 & $0.52-0.6$ (Vert) & $30 \mathrm{~m}$ & $\begin{array}{l}\text { Cartographie de la végétation verte (pic de réflexion de } \\
\text { mesure) ; identification des caractéristiques urbaines et } \\
\text { culturelles. }\end{array}$ \\
\hline
\end{tabular}




\begin{tabular}{|c|l|l|l|}
\hline TM 3 & $\begin{array}{l}0.63-0.69 \\
\text { (Rouge) }\end{array}$ & $30 \mathrm{~m}$ & $\begin{array}{l}\text { La discrimination des espèces végétales et non végétales } \\
\text { (absorption de chlorophylle végétale) ; identification des } \\
\text { caractéristiques urbaines et culturelles. }\end{array}$ \\
\hline TM 4 & $\begin{array}{l}0.76-0.9 \\
\text { (proche Infra } \\
\text { Rouge) }\end{array}$ & $30 \mathrm{~m}$ & $\begin{array}{l}\text { Identification des types de végétation, de la santé et de la } \\
\text { biomasse ; délimitation du corps d'eau ; l'humidité du sol. }\end{array}$ \\
\hline TM 5 & $\begin{array}{l}1.55-1.75 \\
\text { (ondes courtes } \\
\text { UV) }\end{array}$ & $30 \mathrm{~m}$ & $\begin{array}{l}\text { Sensible à l'humidité dans le sol et la végétation ; la neige } \\
\text { exigeante et les zones couvertes par les nuages. }\end{array}$ \\
\hline TM 6 & $\begin{array}{l}10.4-12.5(\text { IR } \\
\text { thermique) }\end{array}$ & $120 \mathrm{~m}$ & $\begin{array}{l}\text { Le stress de la végétation et la discrimination de l'humidité } \\
\text { du sol liés aux rayonnements thermiques ; cartographie } \\
\text { thermique (urbain, eau). }\end{array}$ \\
\hline TM 7 & $\begin{array}{l}2.08-2.35 \\
\text { (ondes courtes } \\
\text { UV) }\end{array}$ & $30 \mathrm{~m}$ & $\begin{array}{l}\text { Discrimination des types de minéraux et de roches ; sensible } \\
\text { à la teneur en humidité de la végétation. }\end{array}$ \\
\hline
\end{tabular}

L'imagerie Landsat 1 a été utilisée pour détecter et cartographier les sommets altérés hydrothermiquement dans une séquence de laves de base dans Hamersley Basra, Western Australia. Les sommets forment des cibles de 1 ou $2 \mathrm{~km}$ de large à cause des creux peu profonds dans la région et peuvent atteindre jusqu'à l'épaisseur réelle des couches altérées qui varient de moins de 5 mètres à environ 30 mètres. Les associations de quartz, d'albite, et de chlorite, dans certains endroits, caractérisent cette altération. Cette dernière a été identifiée à partir d'interprétation d'images EROS en noir et blanc conventionnelles et de couleurs composites de Landsat 1. La disposition de cette altération est bien marquée et a permis de distinguer entre la lave altérée et saine en utilisant des contraintes de contraste de première génération des bandes MSS 4, 5 et 7 agrandies à $1 / 250$ 000, ainsi qu'une composite de couleur de ces bandes. Le rapport de la bande 5 et la bande 7 a permis d'interpréter le produit comme une impression en noir et blanc à une échelle de 1/250 000 . Les essais de classification par ordinateur bloquent une partie de la zone de contrôle et montrent dans certains endroits une amélioration de la discrimination par rapport aux autres affichages (SMITH et al. 1978).

La composite colorée des bandes 1,3 et 5 (bleu, vert et rouge) de l'image satellitaire Landsat 5 du capteur TM (Thematic Mapper) acquise le 28 juin 1986, et après une correction des effets atmosphériques et des distorsions géométriques, et un étalonnage radiométrique du capteur, a permis la réalisation d'une spatiocarte géologique et d'une carte de fracturation des Jebilet centrales ((El Harti et al.2004); (Essaifi et al. 2006) et (El Harti et al. 2006)).

Les bandes 3, 5 et 7 d'ETM+ ont été sélectionnées avec une résolution au sol de $30 \mathrm{~m}$ et une couverture au sol de $185 \times 185 \mathrm{~km}$ au Sudan. L'utilisation de la bande réfléchissante infrarouge moyenne 7, 5 ainsi que la bande 3 dans la lumière visible donnent le meilleur résultat dans la distinction entre le sol 
sec et des surfaces rocheuses pour une variété de formations géologiques (SHADDAD 2005).

L'image Landsat 7 ETM+ a contribué par son apport important à l'élaboration des cartes lithostructurales à la région d'Afara Héouine, Tahifet, Hoggar central, Algérie. Ceci par le biais des différentes compositions colorées, les rapports des bandes, l'analyse en composante principale et les filtres directionnels (matrices $3 \times 3$ ) à $0^{\circ}, 45^{\circ}$ et $90^{\circ}$; selon la méthode illustrée dans la figure 3 (Amriet al., 2009).

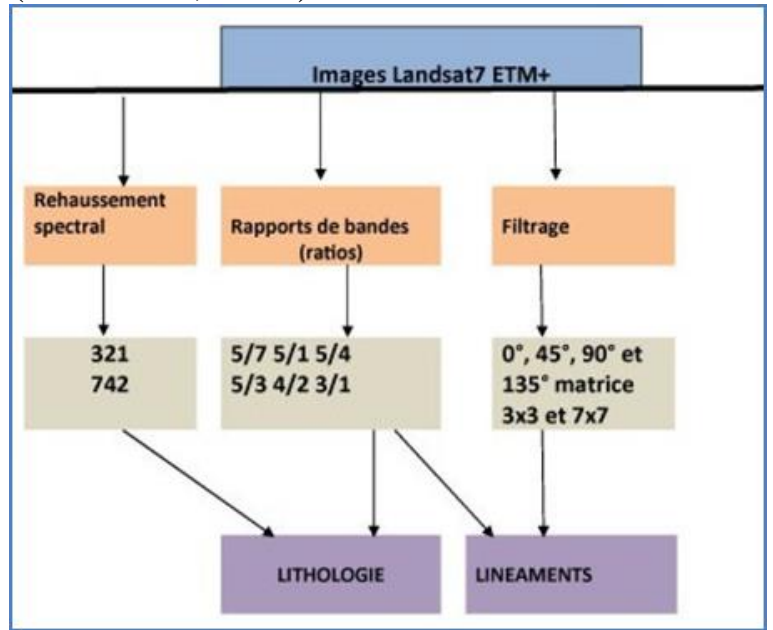

Figure 3 : Méthode de traitement des images Landsat 7 ETM+ à la région d'Afara Héouine, Tahifet, Hoggar central (Amri et al., 2009).

Landsat $7 \mathrm{ETM}+$ a fait l'objet aussi d'un traitement pour étudier sa contribution à la cartographie lithostructurale du Centre-Est de la Cote d'Ivoire (Afrique de l'Ouest) selon la méthode illustrée dans la figure 4. Les filtres directionnels utilisés sont les filtrages de Yesou, Prewitt et Sobel. Le filtre Sobel permet de rehausser les contours mais entraîne un bruitage important. L'application du filtre Prewitt introduit un flou. L'extraction des éléments structuraux a été réalisée grâce à l'application des filtres directionnels sur les bandes ETM+ (Ouattaraet al., 2012). 


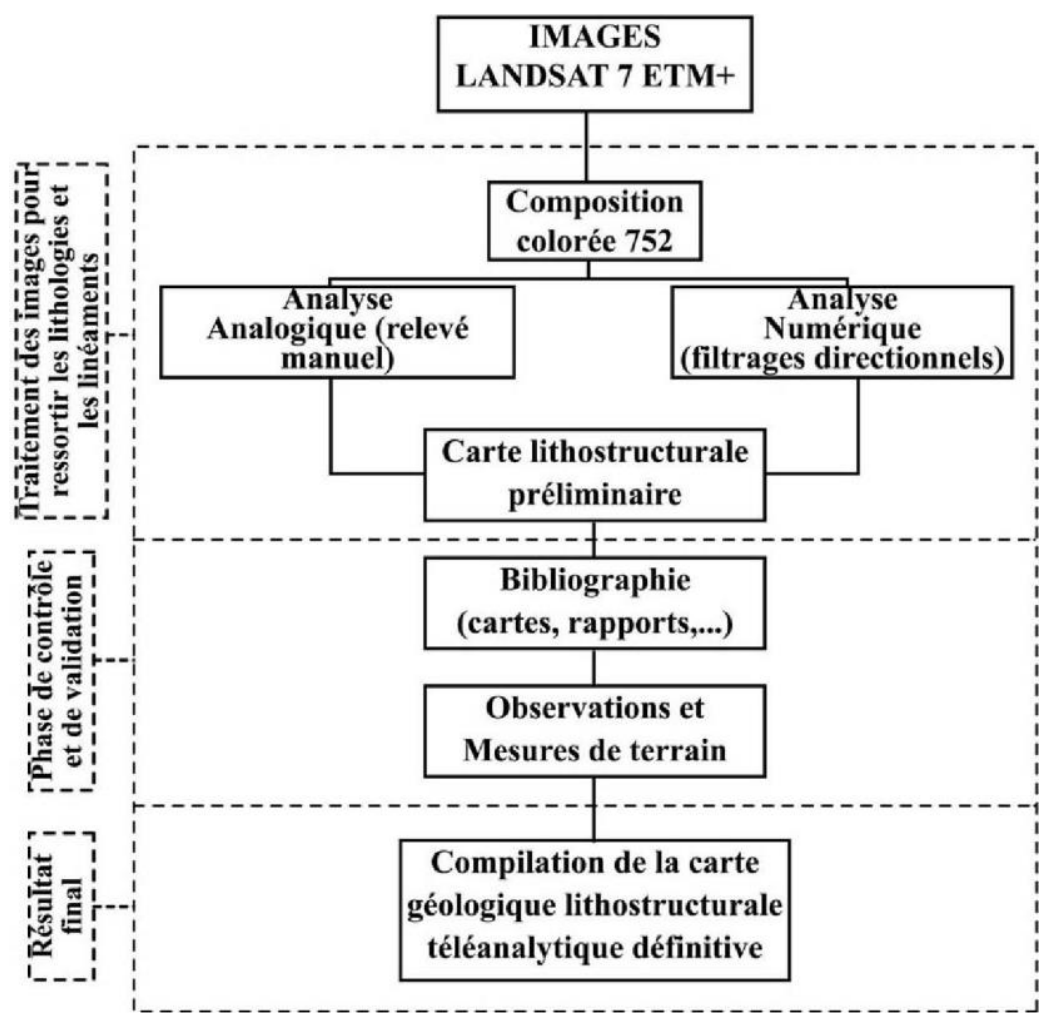

Figure 4 : Chaîne de traitement d'une scène satellite Landsat 7 ETM+ (Ouattara et al.2012).

Le codage des six canaux de même résolution spatiale $30 \mathrm{~m}$ Bandes (1, 2, 3, 4, 5 et 7) d'une image Landsat $7 \mathrm{ETM}+$ avec les trois couleurs fondamentales (rouge, vert et bleu), a permis d'élaborer 216 compositions colorées. Le choix des compositions RVB les plus importantes est effectué à la suite d'une étude statistique pour les six bandes et à la base du coefficient de corrélation des six bandes et leurs déviations standards (Imessaoudene 2012).

Le ratio des bandes est un moyen qui permet de montrer la zone d'altération (Al Hakim \& Sulistijo 2013 ). Le rapport des bandes 5 à réflectance élevée et la bande 7 à faible réflectance montre bien la zone d'altération caractérisée par la présence des minéraux argileux. Les résultats sont visibles sous la forme d'altération avec un net contraste par rapport aux zones environnantes.

Les méthodes standards (Composantes colorées (731 et 742), ACP classique (CP1, CP2 et CP3)) et les méthodes spécifiques (ACP sélectives, les rapports de bandes (5/7 4/5 et 1/3) et (5/3 3/1 et 7/5), transformation RVBITS), classification et filtre appliqué sur ETM+, résumées dans le tableau 2, ont permis une bonne discrimination lithologique entre les formations de la région Hank (Sud-Ouest Algérien) (Achek \& Aidouni 2014). 
Tableau 2: Différentes combinaisons utilisées en fausses couleurs et leurs utilités

(Hamzaoui, 2005).

\begin{tabular}{|c|c|}
\hline RVB & Description \\
\hline 321 & Image en couleur naturelle \\
\hline 432 & Combinaison standard en fausses couleurs. La végétation apparait en rouge. \\
\hline 453 & La limite entre terre / eau est très nette. Bon pour l'étude des sols et de la végétation. \\
\hline 742 & $\begin{array}{l}\text { Combinaison souvent utilisée en géologie. Le sable, le sol et les minéraux montrent une } \\
\text { multitude de couleurs. Bon pour étudier les régions désertiques. }\end{array}$ \\
\hline 753 & $\begin{array}{l}\text { Combinaison souvent utilisée en géologie. Le sable, le sol et les minéraux montrent une } \\
\text { multitude de couleurs. }\end{array}$ \\
\hline 541 & Combinaison souvent utilisée en agronomie. La végétation apparait en vert clair. \\
\hline 754 & $\begin{array}{l}\text { Très utiles en géologie. Fournit la meilleure pénétration dans l'atmosphère. La végétation } \\
\text { apparait en bleu. }\end{array}$ \\
\hline 731 & Combinaison bonne en géologie. Les roches apparaissent dans une multitude de couleurs. \\
\hline
\end{tabular}

Concernant la création des rations, généralement, les bandes de même partie du spectre électromagnétique montrent un «bruit », tandis que les bandes provenant de différentes parties du spectre donnent des bons résultats. Cependant, d'autres applications qu'utilisent des bandes dans la même partie du spectre pour identifier les zones d'altération (MSS : 5/4, 6/5 et 7/6 (4 = vert, $5=$ rouge, $6,7=\mathrm{NIR}) ; \mathrm{TM}: 3 / 2,3 / 1,5 / 7)$. On peut dire que le rapport de deux bandes de la même partie du spectre donne une idée sur la nature globale des objets ( $7 / 3$ lithologie $3 / 5$ neige et glace).

Tableau 3: Utilité des exemples des rapports des bandes (Hamzaoui, 2005).

\begin{tabular}{|c|l|}
\hline Ratio & \multicolumn{1}{c|}{ Description } \\
\hline $5 / 7$ & Bonne discrimination pour les roches riches en Al-OH. \\
\hline $3 / 1$ & $\begin{array}{l}\text { Bonne discrimination pour les roches ayant subies une altération } \\
\text { hydrothermale et pour les oxydes de fer. }\end{array}$ \\
\hline $1 / 2,2 / 1$ & Bonne discrimination pour les roches riches en oxydes de fer. \\
\hline $3 / 1,3 / 2$ & Bonne discrimination de la végétation et l'eau. \\
\hline $4 / 1,4 / 2$ & Bonne discrimination de la végétation et l'eau. \\
\hline $3 / 4$ & Utilisé pour étudier les terres arides et les régions urbaines. \\
\hline $7 / 5$ & Bonne discrimination des argiles. \\
\hline $5 / 4$ & Bonne discrimination des oxydes de fer. \\
\hline
\end{tabular}

Le gisement minier Sar Cheshmeh de cuivre, dans la partie sud-est de la ceinture volcanique Urumieh Dokhtar en Iran, a été choisi comme zone d'étude de cas par M. Pour et M. Hashim. Plusieurs combinaisons en RVB et bandes spécialisées ont été développées à l'aide de bandes Landsat 8, les rapports de bandes obtenus à partir de spectres d'images (4/2, 6/7, 5 et 10 en RVB) permettent l'identification de roches altérées, d'unités lithologiques et de végétation à l'échelle régionale. L'imagerie analytique combinée à la géophysique ont permis de développer des méthodes de traitement des données hyperspectrales. Ces traitements ont été appliqués aux bandes de Landsat-8 pour identifier la zone d'altération associée aux dépôts de porphyre 
cuprifères connus. Dans ce contexte, la méthode de filtrage adaptée par mélange (MTMF) a montré sa grande utilité pour la détection des zones d'altération associées aux dépôts connus de cuivre porphyrique. Des travaux de terrain et des analyses en laboratoire ont été appliquées pour vérifier les résultats de traitement d'image dérivés des bandes Landsat 8. Ces travaux concluent que les bandes Landsat 8, en particulier les bandes 2, 4, 6, 7 et 10 renferment l'information spectrale utile à l'exploration du cuivre porphyrique. De plus, les bandes infrarouges thermiques du Landsat 8 ont amélioré de façon significative la qualité et la disponibilité des données de télédétection infrarouge thermique pour la cartographie lithologique. Ces résultats issus sont d'une grande utilité en l'exploration du cuivre porphyrique dans des contextes similaires (Pour \& Hashim 2014).

\section{Traitement des images Aster :}

L'image Aster joue un rôle important dans la discrimination lithologique. Le tableau 4, donne une idée claire sur 1'utilisation des bandes d'Aster pour l'exploration des substances minérales.

Tableau 4: Les différentes applications des images ASTER (Hamzaoui, 2005).

\begin{tabular}{|c|c|l|}
\hline Bande (Aster) & Résolution & \multicolumn{1}{|c|}{ assemblage et minéraux d'altération } \\
\hline $1,2,3$ & $15 \mathrm{~m}$ & Hématite, jarosite, goethite, ferrihydrite \\
\hline $5(2.145-2.185 \mu \mathrm{m})$ & $30 \mathrm{~m}$ & Kaolinite, dickite, alunite, zunyite, topaz, jarosite, pyrophyllite, \\
\hline $6(2.185-2.225 \mu \mathrm{m})$ & $30 \mathrm{~m}$ & Illite, muscovite, smectite, jarosite \\
\hline $7(2.235-2.285 \mu \mathrm{m})$ & $30 \mathrm{~m}$ & $\begin{array}{l}\text { Chlorite, amphiboles, biotite, épidote, nontronite, tourmaline, } \\
\text { jarosite, dolomite, serpentine }\end{array}$ \\
\hline $8(2.295-2.365 \mu \mathrm{m})$ & $30 \mathrm{~m}$ & $\begin{array}{l}\text { Carbonates, serpentines, nontronite, saponite, chlorite, } \\
\text { amphiboles, biotite, épidote, tourmaline }\end{array}$ \\
\hline $9(2.36-2.43 \mu \mathrm{m})$ & $30 \mathrm{~m}$ & $\begin{array}{l}\text { Chlorite, amphiboles, biotite, épidote, tourmaline, nontronite, } \\
\text { saponite }\end{array}$ \\
\hline
\end{tabular}

Ce satellite a montré son efficacité en matière de prospection minière. Le gisement minier de cuprite, situé dans le centre-ouest du Nevada, est l'un des nombreux centres d'altération explorés pour les métaux précieux. Les roches sédimentaires du Cambrien et les roches volcaniques du Cénozoïque ont été altérées hydrothermiquement par des solutions de sulfate d'acide à faible profondeur durant le Miocène, formant trois assemblages d'altérations :

- Roches silicifiées contenant du quartz et de l'alunite et de la kaolinite rares;

- Roches opalines contenant de l'opale, de l'alunite et de la kaolinite;

- Roches argilisées contenant de la kaolinite et de l'hématite.

Un exemple d'image de l'altération est illustré par la figure 5, en utilisant SWIR d'ASTER et en combinant les bandes 4, 6 et 8 en RVB pour augmenter la saturation des couleurs. 


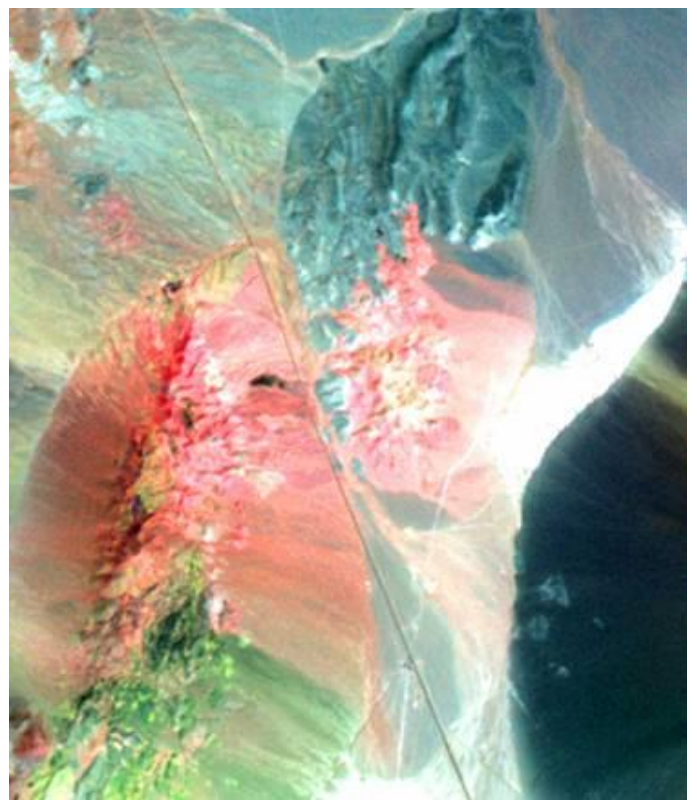

Figure 5 : Image d'Aster, 15 x $20 \mathrm{~km}$, d'un gisement de Cuprite, centre-ouest du Nevada : Visible-proche IR (Mike 2005).

Les zones rouge-rose marquent surtout des roches opalisées avec de la kaolinite et / ou de l'alunite, la zone blanche est Stonewall Playa, les espaces verts sont des calcaires, et les zones bleu-gris sont volcaniques non altérées.

Les données SWIR de la réflectance de surface de la région ont été traitées par le Centre de données EROS (EDC) et les spectres d'image ont été examinés pour des cibles connues. Les données SWIR présentent une diaphonie, ce qui rendait les données difficiles à être utilisées en analyse spectrale en comparaison directe avec des spectres de bibliothèque ou de terrain. Pour réduire les artefacts de diaphonie, un spectre de Stonewall Playa a été utilisé comme cible, rééchantillonné aux longueurs d'onde ASTER et divisé en données SWIR de réflectance. Des spectres de bibliothèque ont été compilés pour les minéraux connus à cuprite; Ils ont été ensuite rééchantillonnés à des longueurs d'onde SWIR d'ASTER. Ces spectres ont été utilisés avec un algorithme de classification supervisé pour cartographier des occurrences spectrales similaires dans les données SWIR qui identifient les différentes classes de l'image sur la base du calcul de l'angle spectral (Spectral Angle Mapper). Le résultat de cette classification est illustré à la figure 6 . L'angle spectral est calculé entre le vecteur de test construit pour chaque pixel et le vecteur de référence construit pour chaque classe de référence sélectionnée par l'utilisateur. Les résultats obtenus permettent de lire et de réorganiser de multiples ensembles de données en 2 dimensions en un unique cube compact de données 3D. Le vecteur de référence est construit pour effectuer la classification SAM et l'angle entre le vecteur de référence et le 
vecteur de pixel est calculé pour comparer avec la valeur d'angle de seuil déterminée. Le codage couleur est ensuite appliqué pour distinguer entre les différentes classes qui ont été reconnues par l'algorithme SAM. Ainsi, en utilisant ce dernier, des images hyperspectrales sont analysées pour en ressortir toutes informations thématiques utiles telles que la couverture du sol, les masses d'eau et les nuages (Rashmi et al. 2014).

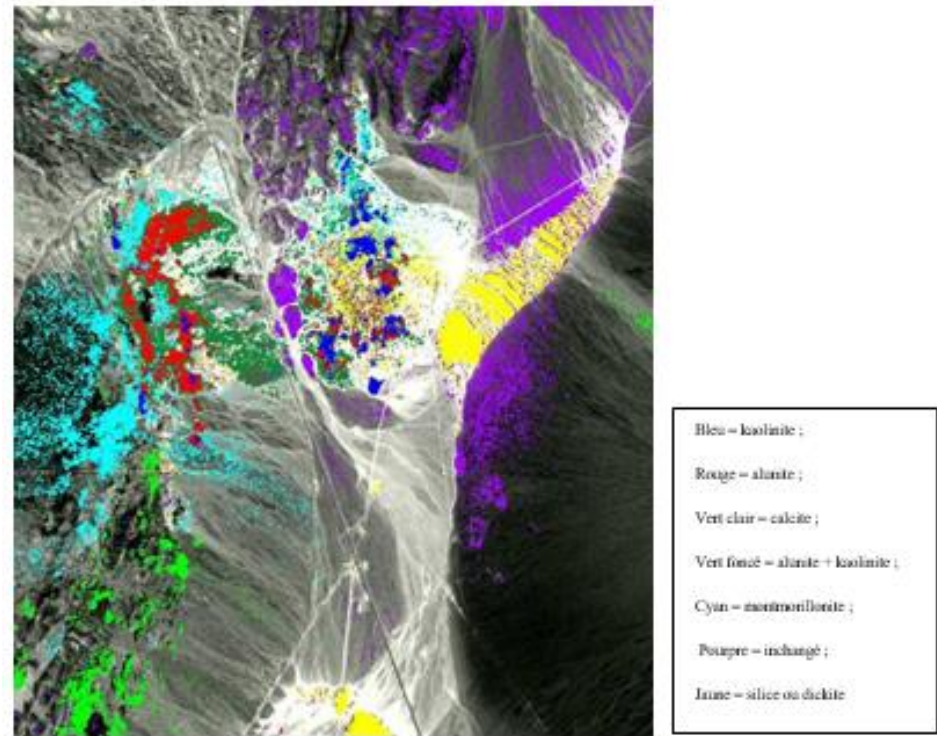

Figure 6 : Classification par angle angulaire spectral des données SWIR de Cuprite.

La figure 7 présente aussi le centre ouest du Nevada avec une composante différente des couleurs Rouge-vert-Bleu (RVB).

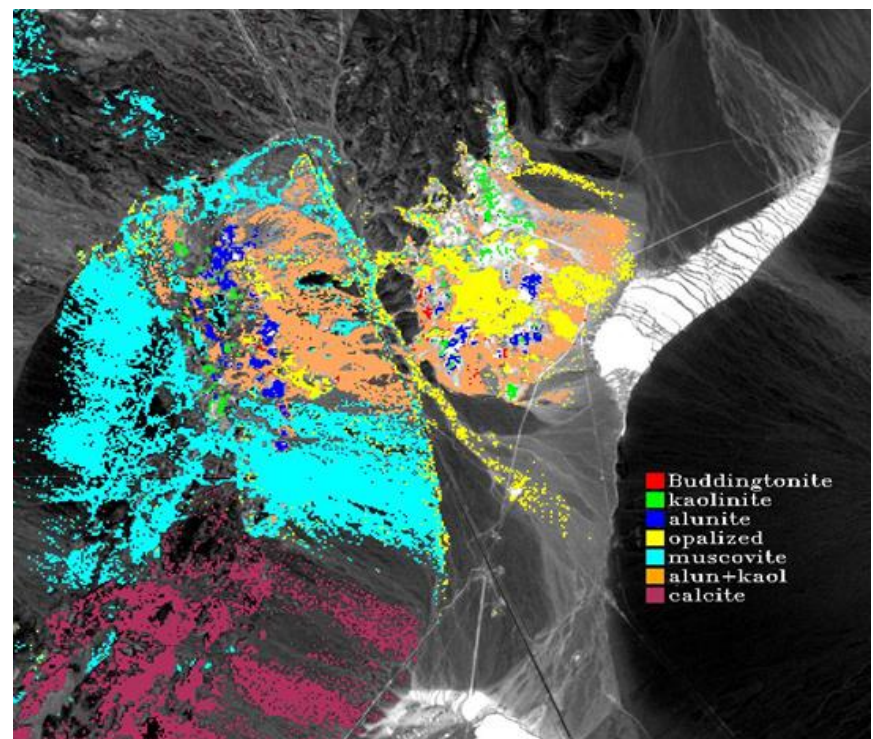

Figure 7 : Les données SWIR d'ASTER pour la classification du Cuprite en utilisant Spectral Angle Mapper (Mike, 2005). 
La comparaison de la présente carte à une classification minérale plus détaillée produite à partir de données AVIRIS, a permis de constater une excellente correspondance. Les spectres de bibliothèque rééchantillonnés sont représentés sur la figure 8, comparativement aux spectres d'image ASTER extraits de zones de $3 \times 3$ pixels.
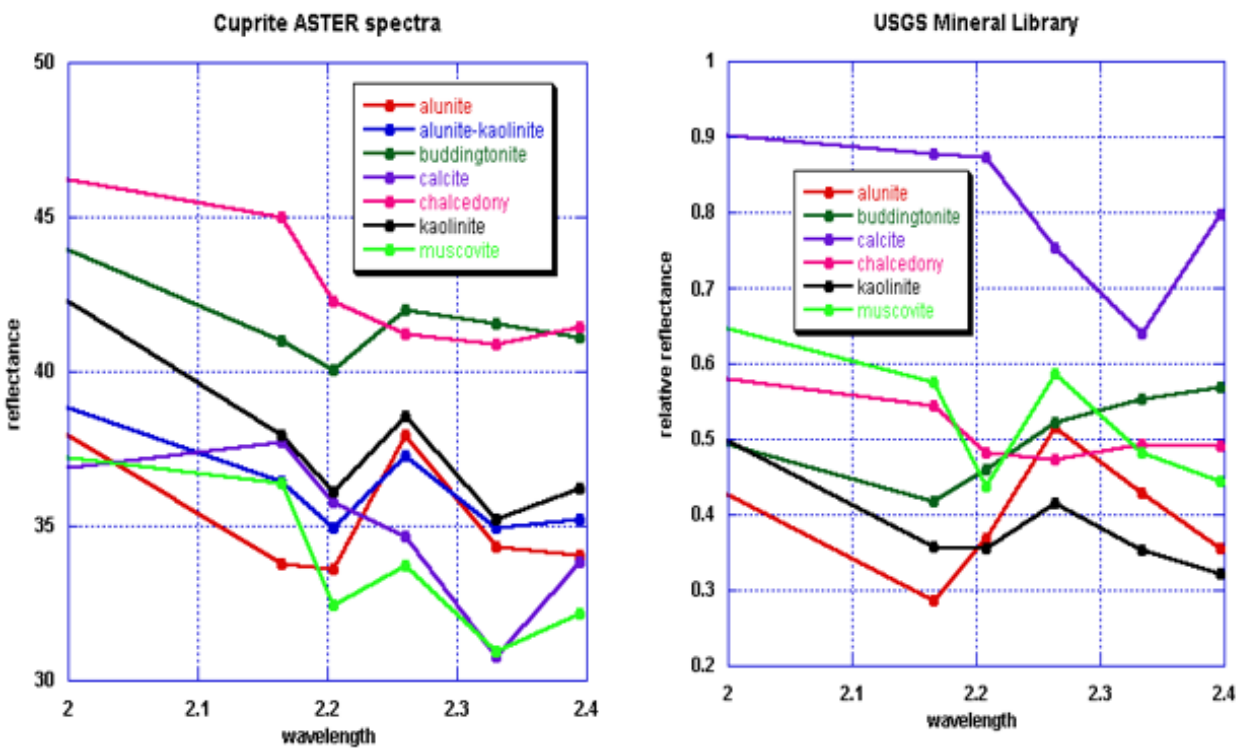

Figure 8: Spectres SWIR des minéraux cartographiés à Cuprite : Image ASTER (à gauche) \& Bibliothèque USGS (à droite) (Mike, 2005)

L'image SWIR d'ASTER de l'Ouest-centre du Nevada, représentée par la figure 9, présente une composante des bandes 4-6-8 RVB.

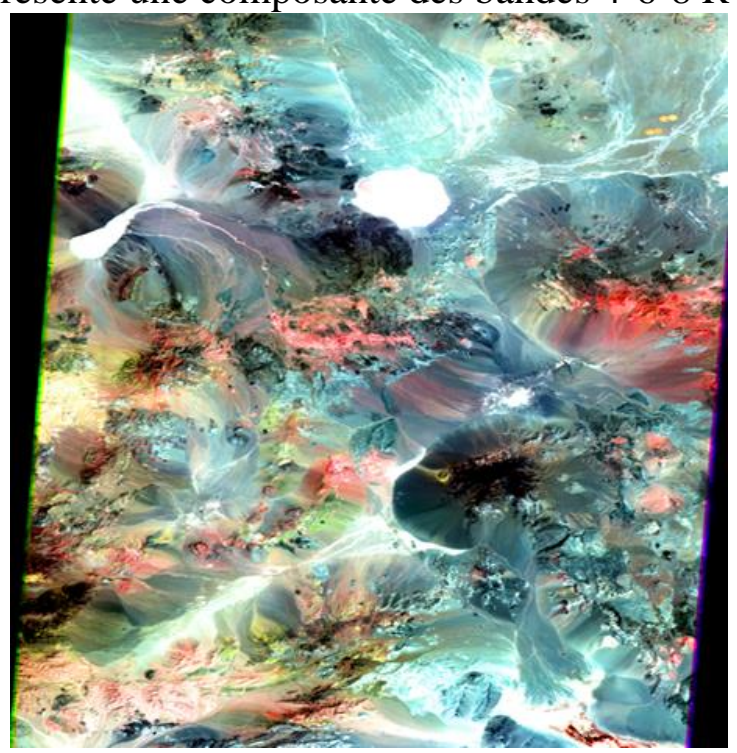

Figure 9 : Image SWIR d'ASTER avec une composante des bandes 4-6-8 RVB de l'Ouestcentre du Nevada (Mike, 2005). 
Les montagnes de l'Anti-Atlas du Maroc se sont formées à la suite de la collision des plaques tectoniques africaines et eurasiennes il y a environ 80 millions d'années. Cette collision a détruit l'océan Téthys. Les couches de calcaire, de grès, de pierre d'argile et de gypse formant le lit de l'océan ont été pliées et froissées pour créer les Montagnes de l'Anti-Atlas. Dans la figure 10, des bandes infrarouges à courte longueur d'onde (bande 4 à 9), sont combinées pour mettre en évidence de façon spectaculaire les différentes types de roches et illustrer le pliage complexe des formations géologiques. Les zones jaunâtres, oranges et vertes présentent des calcaires, des grès et du gypse ; Les zones bleu foncé et vertes sont des roches granitiques sous-jacentes.

La capacité de cartographier la géologie à l'aide de données ASTER est renforcée par les bandes SWIR multiples, qui sont sensibles aux différences dans la minéralogie des roches. L'image, figure 10, a été acquise le 13 juin 2001, couvre une superficie de 28,7 x 29,4 km, et est située à 29,4 degrés de latitude nord et 8,9 degrés de longitude ouest (NASA, METI, AIST, Systems, \& Team).

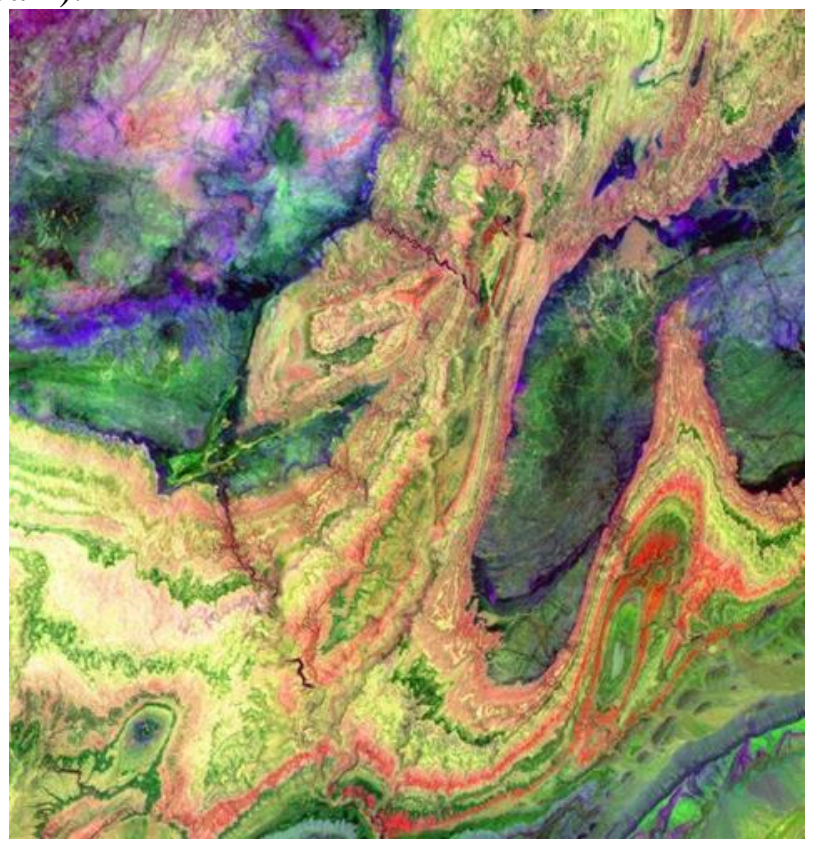

Figure 10 : Image ASTER de l'Anti Atlas.

Les données ASTER ont été appliquées pour l'exploration des gisements miniers, comme le gisement de Ni-Cu dans l'est de Tian Shan, le gypse dans l'ouest de Tian Shan, Asie centrale . La méthodologie d'extraction d'informations depuis les images ASTER donne des bons résultats en ce qui concerne la détection des anomalies pour le dépôt de $\mathrm{Ni}-\mathrm{Cu}$, le gypse et le borax (Zhang \& Yao 2015). 


\section{Traitement des images couplé avec d'autres techniques :}

Pour un choix réellement quantitatif des meilleures combinaisons de bandes pour l'extraction d'un maximum d'information, Chavez et al, (1982) ont introduit la notion d'Optimum Index Factor «OIF ». Cet indice est une valeur statistique utilisée pour sélectionner la combinaison optimale de trois bandes dans une image satellitaire afin de créer une composite. La combinaison optimale de bandes parmi toutes les combinaisons possibles à 3 bandes présente le plus grand nombre d'informations (= la somme la plus élevée des écarts types), avec le moins de duplication (la plus faible corrélation entre les paires de bandes) (spatial-analyst.net). L'OIF est calculé selon l'équation suivante :

$$
O I F=\frac{\sum_{K=1}^{3} S_{K}}{\sum_{j=1}^{3}|\Gamma j|}
$$

Équation 1: Optimum Index Factor (Chavez et al.1982).

Où

$\Sigma \mathrm{Sk}=$ Somme des écarts types (déviations standards) des combinaisons des 3 bandes k1, k 2, k 3 .

$\Sigma / \mathrm{rj} /=$ Somme des valeurs absolues du coefficient de corrélation.

La combinaison dont l'OIF le plus élevé est susceptible de fournir le maximum d'informations lithologiques puisqu'elle utilise les données ayant le moins de redondances.

Le but est d'utiliser la technique OIF pour classer toutes les combinaisons possibles de couleurs de bandes de données ETM + pour la cartographie. La technique OIF a été appliquée à toutes les bandes de données Enhanced Thematic Mapper Plus (ETM +) du Landsat 7. Un certain nombre de combinaisons de couleurs à 56 bandes ont été produites et analysées en utilisant l'OIF. Les différentes analyses de toutes les valeurs de l'OIF et de leurs rangs ont montré que la technique de l'OIF est utile pour sélectionner la combinaison appropriée pour la cartographie géologique de la zone d'étude. L'utilisation de la technique OIF pour l'évaluation des bandes de données ETM + a simplifié la sélection de bandes appropriées pour discriminer les différentes unités de roches. Il réduit également le temps de sélection. L'ajout de la bande panchromatique de $15 \mathrm{~m}$ de résolution de ETM + au VNIR, SWIR et TIR a amélioré leur résolution et a facilité également les interprétations géologiques. La valeur la plus élevée de l'OIF est 45.65 qui correspondent à la combinaison des bandes 5, 6 et 7 (Qaid \& Basavarajappa 2008).

La cartographie lithologique est l'une des étapes fondamentales des différentes études de prospection minière, car elle constitue la base de l'interprétation et de la validation des résultats extraits. Par conséquent, on peut 
exploiter les données multispectrales ASTER et Landsat 8 afin de cartographier les unités lithologiques dans la boutonnière de Bas Drâa à l'Anti Atlas occidental. Cette tâche a été complétée par l'utilisation de l'analyse des composantes principales $(\mathrm{ACP})$, rapport des bandes $(\mathrm{BR})$ et de la Machine à vecteurs de support (SVM). La précision globale et le coefficient kappa des SVM basés sur la vérité au sol, en plus des résultats d'ACP et BR, montrent une excellente corrélation avec les cartes géologiques existantes et les travaux antérieurs sur la zone d'étude. Par conséquent, la méthodologie proposée démontre un potentiel élevé de données ASTER et Landsat 8 dans la discrimination des unités lithologiques (Adiri et al.2016).

Selon Dabo \& Aïfa,(2013), les linéaments ont été cartographiés à partir $\mathrm{du}$ traitement de plusieurs types d'images selon une approche multidisciplinaire :

- La combinaison des bandes 4.3.2, TM et ETM7+, qui atténue les effets des nuages et met la végétation en bleu grisâtre, les sols latéritiques en jaune et l'eau en bleu noirâtre; et la combinaison des bandes 2.3.1 qui fait apparaître la végétation en cyan et bleu sombre, l'eau en blanc et les sols latéritiques en rouge. Il est intéressant de signaler que la bande panchromatique (bande 8 ) présente une meilleure résolution pour la visualisation et l'extraction des linéaments.

- L'image SRTM (Shuttle Radar Topography Mission) ou MNT (Modèle Numérique de Terrain) de la navette spatiale Endeavour a été utilisée pour corriger les effets des ombrages directionnels ;

- L'image aéromagnétique de la zone d'étude. Le résultat issu du traitement de ces données géophysiques met en évidence les structures géologiques de sub-surface.

La fusion d'images optiques et celle de radar présente un grand intérêt pour la recherche qui nécessite une très grande investigation liée au domaine de l'informatique. En géologie, étant les images optiques multispectrales et hyperspectrales permettent de déterminer la lithologie sur la base des signatures spectrales des cibles, les images radar, grâce à leur grande sensibilité à la rugosité de surface, sont les plus adéquates pour la cartographie structurale.

Cette technique de fusion d'images ITS (Intensité, Teinte, Saturation) semble plus performante que l'analyse en composantes principales (ACP) et donne la possibilité de pondérer la contribution du radar dans l'image résultante de la fusion des données optiques SPOT HRV-XS, Landsat-TM et du radar ERS-2. L'application des méthodes de classification en deux étapes, d'abord, par classification bayésienne dirigée sur une image Landsat-TM et sur une image Radar ERS-2, puis par fusion radar-optique des résultats de ces 
deux classifications, ont permis de compléter l'interprétation dans la zone d'étude (HAKDAOU et al. 2005).

\section{Méthodes de traitement et proposition de modèle adéquat :}

L'exploration minière constitue une phase préliminaire de toute activité d'exploitation. Toutefois, elle a connu une évolution remarquable liée au développement des nouveaux outils et approches surtout dans les sites et des terrains étudiés inaccessibles.

Après avoir présenté, dans l'état de l'art, les différentes méthodes et approches réalisées par plusieurs spécialistes dans ce domaine de recherche qui définissent de près l'utilisation de la télédétection pour la cartographie lithostructurale et minéralogique, en général, et l'exploration et la recherche des substances minérales, en particulier. Le but principal de cette partie est de 1) créer des méthodes dérivées à partir des méthodes de traitement utilisées et mentionnées dans l'état de l'art, au-dessus, par le biais d'une analogie logique entre les différentes bandes de plusieurs satellites d'observation du globe terrestre notamment entre : Landsat 7 ETM+ ; Landsat 8 OLI /TIRS ; Aster et Sentinel 2A, 2) synthétiser ces résultats par la proposition d'un modèle de traitement des images multispectrales qui peut être appliqué directement sur les différentes zones d'études pour la cartographie lithostructurale et minéralogique.

\subsection{Méthodes de traitement utilisées dans l'état de l'art et leurs méthodes dérivées.}

Il s'est avéré que plusieurs études utilisent les données Landsat 7 ETM+ vu utilité de ces données pour la discrimination géologique. On va les utiliser soit en les appliquant directement sur notre zone d'étude, l'Anti-Atlas central et occidental, soit en adoptant des nouvelles méthodes de traitement pour répondre à notre problématique (l'exploration minière via les images satellitaires). Le tableau 5 et la figure 11 présentent les différences entre image satellitaire du Landsat 7 ETM+ et Landsat 8 OLI/TIRS.

Tableau 5: Comparaison entre Bandes spectrales ETM + et OLI / TIRS (USGS, 2010).

\begin{tabular}{|l|l|l|}
\hline \multicolumn{1}{|c|}{ Bandes L7 ETM+ } & \multicolumn{1}{|c|}{$\begin{array}{c}\text { Bandes L8 LDCM OLI / } \\
\text { TIRS }\end{array}$} & \multicolumn{1}{|c|}{ Explication des différences } \\
\hline & $\begin{array}{l}\text { Bande } 1: 30 \mathrm{~m} \text {, Coastal / } \\
\text { Aerosol, } 0.433-0.453 \mu \mathrm{m}\end{array}$ & $\begin{array}{l}\text { Bande côtière ajoutée à la demande des chercheurs } \\
\text { de couleur océanique exigeant une résolution plus } \\
\text { élevée des eaux côtières par rapport à MODIS et } \\
\text { SeaWiFS. }\end{array}$ \\
\hline Bande $1: 30 \mathrm{~m}$, Blue, & Bande $2: 30 \mathrm{~m}$, Blue, \\
$0.450-0.515 \mu \mathrm{m}$ & $0.450-0.515 \mu \mathrm{m}$ & \\
\hline Bande $2: 30 \mathrm{~m}$, Green, & Bande $3: 30 \mathrm{~m}$, Green, \\
$0.525-0.605 \mu \mathrm{m}$ & $0.525-0.600 \mu \mathrm{m}$ & \\
\hline
\end{tabular}




\begin{tabular}{|c|c|c|}
\hline $\begin{array}{l}\text { Bande 3: } 30 \mathrm{~m}, \text { Red, } \\
0.630-0.690 \mu \mathrm{m}\end{array}$ & $\begin{array}{l}\text { Bande } 4: 30 \mathrm{~m}, \text { Red, } \\
0.630-0.680 \mu \mathrm{m}\end{array}$ & \multirow{5}{*}{$\begin{array}{l}\text { Amélioration de la bande passante effectuée pour } \\
\text { éviter les caractéristiques d'absorption } \\
\text { atmosphérique (activée par le rapport signal-tonoise } \\
\text { plus élevé inhérent à l'architecture push-broom) }\end{array}$} \\
\hline $\begin{array}{l}\text { Bande } 4: 30 \mathrm{~m}, \text { Near- } \\
\text { IR, } 0.775-0.900 \mu \mathrm{m}\end{array}$ & $\begin{array}{l}\text { Bande } 5: 30 \mathrm{~m} \text {, Near-IR, } \\
0.845-0.885 \mu \mathrm{m}\end{array}$ & \\
\hline $\begin{array}{l}\text { Bande 5: } 30 \mathrm{~m}, \text { SWIR- } \\
1,1.550-1.750 \mu \mathrm{m}\end{array}$ & $\begin{array}{l}\text { Bande } 6: 30 \mathrm{~m}, \text { SWIR-1, } \\
1.560-1.660 \mu \mathrm{m}\end{array}$ & \\
\hline $\begin{array}{l}\text { Bande } 7: 30 \mathrm{~m}, \text { SWIR- } \\
2,2.090-2.350 \mu \mathrm{m}\end{array}$ & $\begin{array}{l}\text { Bande } 7: 30 \mathrm{~m}, \text { SWIR-2, } \\
2.100-2.300 \mu \mathrm{m}\end{array}$ & \\
\hline \multirow[t]{2}{*}{$\begin{array}{l}\text { Bande } 8: 15 \mathrm{~m}, \text { Pan, } \\
0.520-0.900 \mu \mathrm{m}\end{array}$} & $\begin{array}{l}\text { Bande } 8: 15 \mathrm{~m}, \text { Pan } \\
0.500-0.680 \mu \mathrm{m}\end{array}$ & \\
\hline & $\begin{array}{l}\text { Bande } 9: 30 \mathrm{~m} \text {, Cirrus, } \\
1.360-1.390 \mu \mathrm{m}\end{array}$ & $\begin{array}{l}\text { Bande de cirrus ajoutée pour détecter la } \\
\text { contamination des nuages dans d'autres canaux. }\end{array}$ \\
\hline \multirow[t]{2}{*}{$\begin{array}{l}\text { Bande 6: } 60 \mathrm{~m}, \mathrm{LWIR}, \\
10.00-12.50 \mu \mathrm{m}\end{array}$} & $\begin{array}{l}\text { Bande } 10: 100 \mathrm{~m}, \text { LWIR- } \\
1,10.30-11.30 \mu \mathrm{m}\end{array}$ & \multirow{2}{*}{$\begin{array}{l}\text { TIRS acquerra les données pour ces deux bandes } \\
\text { thermiques. }\end{array}$} \\
\hline & $\begin{array}{c}\text { Bande } 11: 100 \mathrm{~m}, \text { LWIR- } \\
2,11.50-12.50 \mu \mathrm{m}\end{array}$ & \\
\hline
\end{tabular}

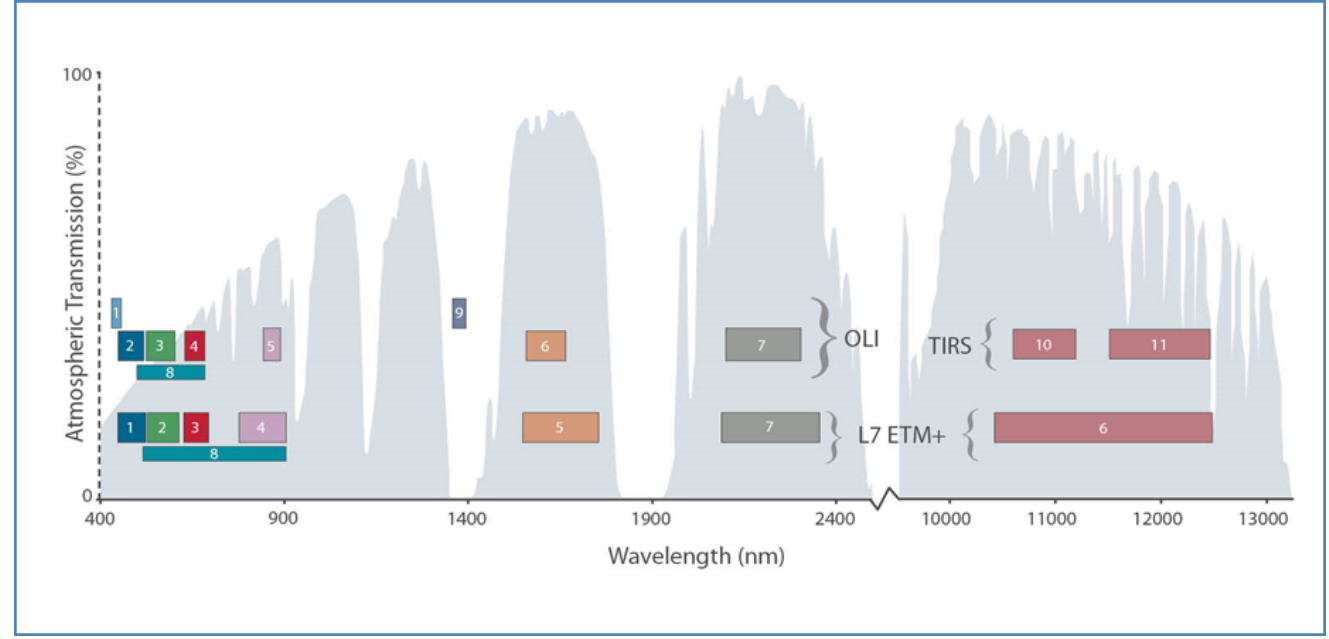

Figure 11 : Les différences entre image satellitaire du Landsat 7 ETM+ et Landsat 8 OLI/TIRS (NASA, Landsat).

Grâce à une analogie entre les bandes des deux instruments ETM + et OLI / TIRS, on peut créer une nouvelle méthode de traitement basé sur la méthode de traitement d'images ETM + réalisé par (Amri et al.2009). Le schéma de la figure 12 illustre notre nouvelle méthode. 


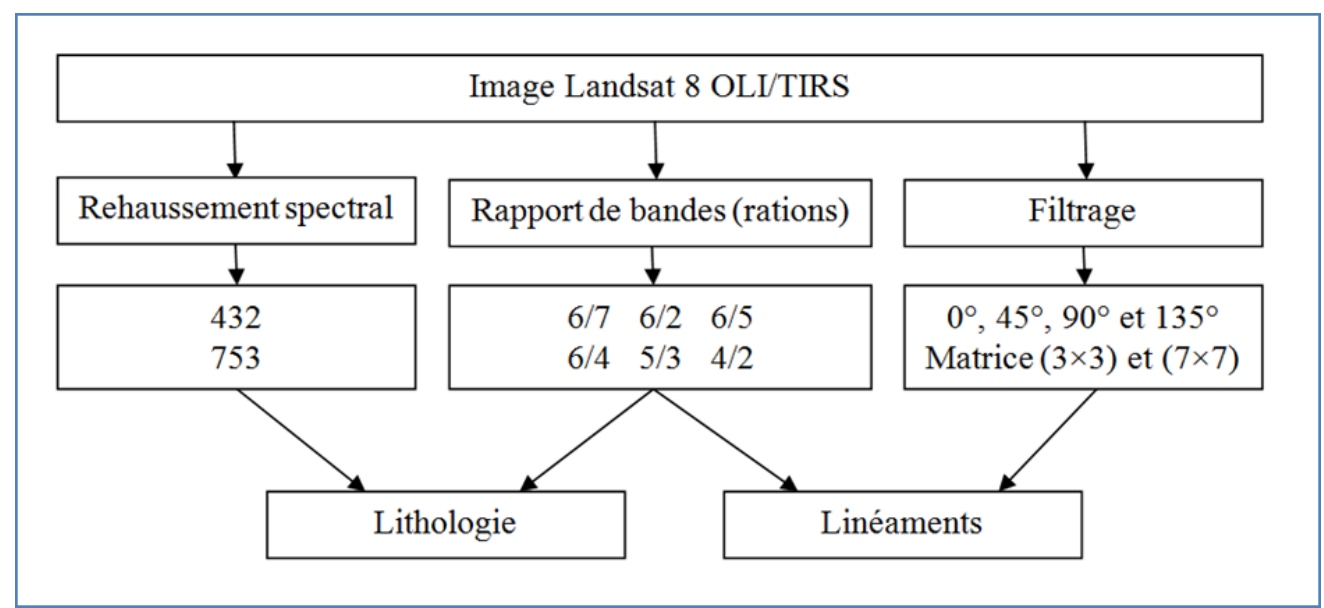

Figure 12: Méthode de traitement des données Landsat 8.

De la même façon, on peut créer plusieurs méthodes par analogique (tableau 6), surtout que l'utilisation des données d'OLI / TIRS est plus efficace que les données ETM +.

Tableau 6: Combinaisons colorées et rapport des bandes de ETM + et d'OLI/TIRS.

\begin{tabular}{|c|c|c|}
\hline $\begin{array}{l}\text { Combinaisons } \\
\text { colorées et rapport } \\
\text { des bandes de } \\
\text { ETM + }\end{array}$ & $\begin{array}{l}\text { Combinaisons colorées et } \\
\text { rapport des bandes d'OLI / } \\
\text { TIRS crées par analogie }\end{array}$ & Utilité \\
\hline 321 & 432 & Vision naturelle \\
\hline 752 & 763 & Carte lithostructurale \\
\hline 432 & 543 & $\begin{array}{l}\text { Discrimination lithologique, la } \\
\text { végétation apparait en rouge. }\end{array}$ \\
\hline 231 & 342 & \multirow{7}{*}{ Discrimination lithologique } \\
\hline 731 & 742 & \\
\hline 742 & 753 & \\
\hline $5 / 74 / 51 / 3$ & $6 / 75 / 62 / 4$ & \\
\hline $5 / 33 / 17 / 5$ & $6 / 44 / 27 / 6$ & \\
\hline $5 / 75 / 15 / 4$ & $6 / 76 / 26 / 5$ & \\
\hline $5 / 34 / 23 / 1$ & $6 / 45 / 34 / 2$ & \\
\hline 753 & 764 & $\begin{array}{l}\text { Délimitation du sol sec et des surfaces } \\
\text { rocheuses distincte }\end{array}$ \\
\hline 754 & 765 & $\begin{array}{l}\text { Discrimination lithologique, la } \\
\text { végétation apparait en bleu. }\end{array}$ \\
\hline $3 / 1$ & $4 / 2$ & $\begin{array}{l}\text { Bonne discrimination pour les roches } \\
\text { ayant subies une altération } \\
\text { hydrothermale et pour les Oxydes de } \\
\text { Fer. }\end{array}$ \\
\hline $1 / 2,2 / 1$ & $2 / 3,3 / 2$ & $\begin{array}{l}\text { Bonne discrimination pour les roches } \\
\text { riches en Oxydes de Fer. }\end{array}$ \\
\hline $5 / 7$ & $6 / 7$ & $\begin{array}{l}\text { Bonne discrimination pour les roches } \\
\text { riches en Al-OH. }\end{array}$ \\
\hline
\end{tabular}




\begin{tabular}{|l|l|l|}
\hline $7 / 5$ & $7 / 6$ & Bonne discrimination des argiles. \\
\hline $5 / 4$ & $6 / 5$ & $\begin{array}{l}\text { Bonne discrimination des oxydes de } \\
\text { Fer. }\end{array}$ \\
\hline
\end{tabular}

On peut aussi utiliser une analogie entre les données des satellites ETM+, OLI/TIRS, Sentinel 2A et Aster grâce aux figures 13 et 14.

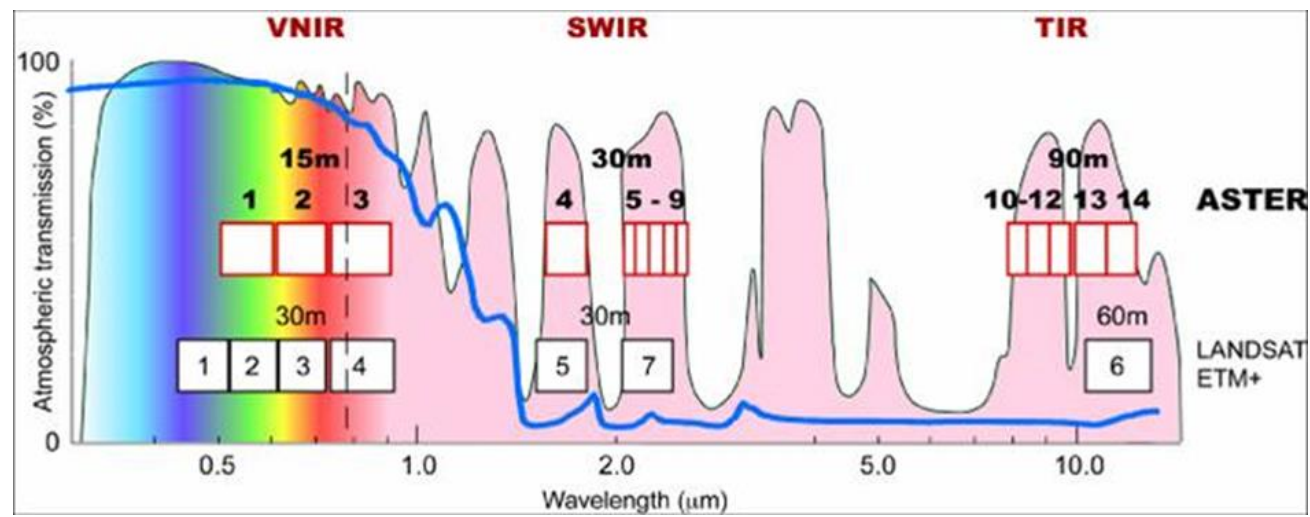

Figure 13 : Comparaison entre les données ETM+ et les données Aster.

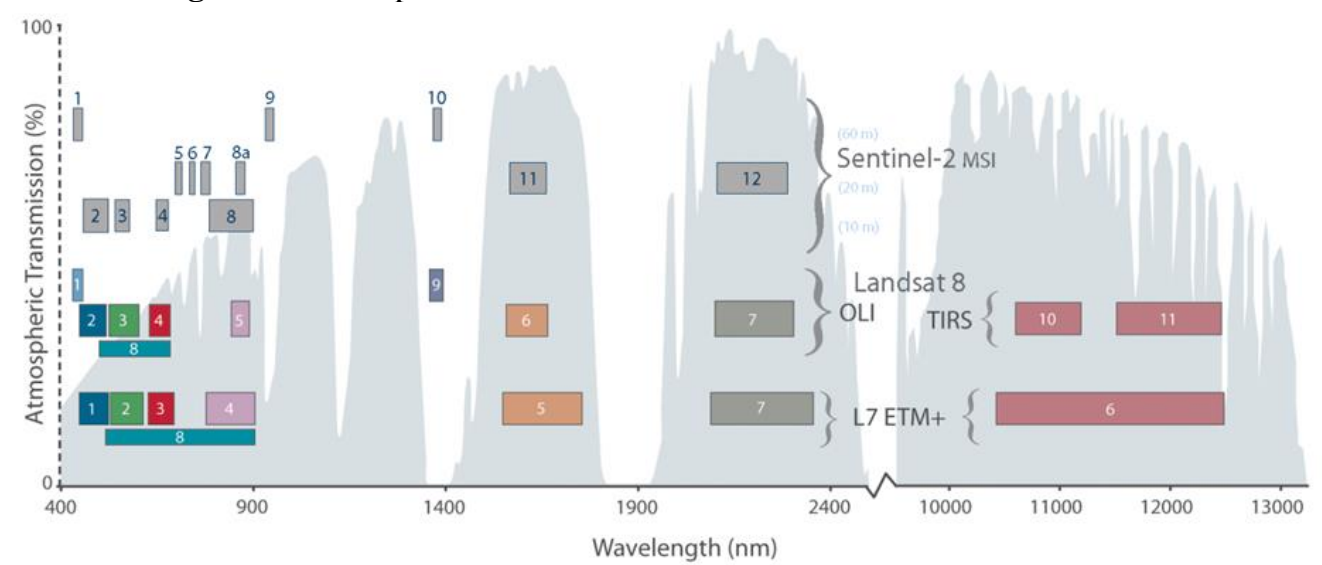

Figure 14 : Les différences entre les images satellitaires du Landsat (7 ETM+ et 8 OLI/TIRS) et Sentinel 2A MSI (NASA).

De la même façon, la création d'une analogie entre les données d'ETM+, Landsat 8 et autres satellites peut se faire grâce à une correspondance des bandes présentées dans le tableau 7.

Tableau 7: Correspondance les bandes entre les satellites (L7 ETM+; Landsat 8; Sentinel 2A et Aster).

\begin{tabular}{|l|l|l|l|}
\hline Bandes L7 ETM+ & Bandes L8 LDCM OLI / TIRS & Sentinel 2 A & Aster \\
\hline- & Bande $1: 30 \mathrm{~m}$, Coastal /Aerosol, & \multirow{2}{*}{ B01 : 60 m } & - \\
\hline Bande $1: 30 \mathrm{~m}$, Blue, & $0.433-0.453 \mu \mathrm{m}$ & & \\
$0.450-0.515 \mu \mathrm{m}$ & Bande $2: 30 \mathrm{~m}$, Blue, 0.450- & B2:10 m $0.4575-$ & - \\
\hline Bande $2: 30 \mathrm{~m}$, Green, & $0.515 \mu \mathrm{m}$ & 0.5225 & - \\
$0.525-0.605 \mu \mathrm{m}$ & $0.600 \mu \mathrm{m}$ & B3:10 $\mathrm{m} 0.5425-$ & Bande 1: VNIR 15 m \\
\hline
\end{tabular}




\begin{tabular}{|c|c|c|c|}
\hline $\begin{array}{l}\text { Bande 3: } 30 \mathrm{~m} \text {, Red, } 0.630- \\
0.690 \mu \mathrm{m}\end{array}$ & $\begin{array}{l}\text { Bande } 4: 30 \mathrm{~m}, \text { Red, } 0.630- \\
0.680 \mu \mathrm{m}\end{array}$ & $\begin{array}{l}\text { B4:10 m } 0.65- \\
0.68\end{array}$ & $\begin{array}{l}\text { Bande 2: VNIR } 15 \mathrm{~m} \\
0.63-0.69 \mu \mathrm{m}\end{array}$ \\
\hline $\begin{array}{l}\text { Bande } 4: 30 \mathrm{~m} \text {, Near-IR, } \\
0.775-0.900 \mu \mathrm{m}\end{array}$ & $\begin{array}{l}\text { Bande } 5: 30 \mathrm{~m} \text {, Near-IR, } 0.845- \\
0.885 \mu \mathrm{m}\end{array}$ & $\begin{array}{l}\text { B8: } 10 \mathrm{~m} 0.7845- \\
0.8995 \\
\text { B08A : } 20 \mathrm{~m}\end{array}$ & $\begin{array}{l}\text { Bande 3: VNIR } 15 \mathrm{~m} \\
0.76-0.86 \mu \mathrm{m}\end{array}$ \\
\hline $\begin{array}{l}\text { Bande 5: } 30 \mathrm{~m}, \mathrm{SWIR}-1, \\
1.550-1.750 \mu \mathrm{m}\end{array}$ & $\begin{array}{l}\text { Bande } 6: 30 \mathrm{~m}, \text { SWIR-1, } 1.560- \\
1.660 \mu \mathrm{m}\end{array}$ & $\begin{array}{l}\text { B11: } 1.565- \\
1.655\end{array}$ & $\begin{array}{l}\text { Bande 4: SWIR 30m } \\
1.600-1.700 \mu \mathrm{m}\end{array}$ \\
\hline \multirow{2}{*}{$\begin{array}{l}\text { Bande 6: } 60 \mathrm{~m}, \mathrm{LWIR} \text {, } \\
10.00-12.50 \mu \mathrm{m}\end{array}$} & \multirow{2}{*}{$\begin{array}{l}\text { Bande } 10: 100 \mathrm{~m}, \text { LWIR-1, } \\
10.30-11.30 \mu \mathrm{m}\end{array}$} & \multirow[t]{2}{*}{ 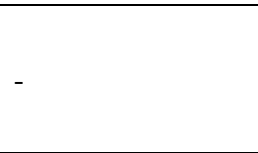 } & $\begin{array}{l}\text { Bande 13: TIR 90m } \\
10.25-10.95 \mu \mathrm{m}\end{array}$ \\
\hline & & & $\begin{array}{l}\text { Bande 14: TIR 90m } \\
10.95-11.65 \mu \mathrm{m}\end{array}$ \\
\hline \multirow{4}{*}{$\begin{array}{l}\text { Bande } 7: 30 \mathrm{~m}, \text { SWIR-2, } \\
2.090-2.350 \mu \mathrm{m}\end{array}$} & \multirow{4}{*}{$\begin{array}{l}\text { Bande } 7: 30 \mathrm{~m}, \text { SWIR-2, } 2.100- \\
2.300 \mu \mathrm{m}\end{array}$} & \multirow{4}{*}{ B12: $2.1-2.28$} & $\begin{array}{l}\text { Bande 5: SWIR 30m } \\
2.145-2.185 \mu \mathrm{m}\end{array}$ \\
\hline & & & $\begin{array}{l}\text { Bande 6: SWIR 30m } \\
2.185-2.225 \mu \mathrm{m}\end{array}$ \\
\hline & & & $\begin{array}{l}\text { Bande 7: SWIR 30m } \\
2.235-2.285 \mu \mathrm{m}\end{array}$ \\
\hline & & & $\begin{array}{l}\text { Bande 8: SWIR 30m } \\
2.295-2.365 \mu \mathrm{m}\end{array}$ \\
\hline $\begin{array}{l}\text { Bande 8: } 15 \mathrm{~m}, \text { Pan, } 0.520- \\
0.900 \mu \mathrm{m}\end{array}$ & $\begin{array}{l}\text { Bande } 8: 15 \mathrm{~m}, \text { Pan } 0.500-0.680 \\
\mu \mathrm{m}\end{array}$ & - & - \\
\hline 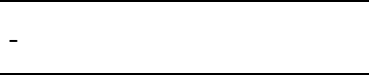 & $\begin{array}{l}\text { Bande } 9: 30 \mathrm{~m} \text {, Cirrus, } 1.360- \\
1.390 \mu \mathrm{m}\end{array}$ & $\mathrm{B} 10: 60 \mathrm{~m}$ & - \\
\hline- & $\begin{array}{l}\text { Bande } 11: 100 \mathrm{~m}, \mathrm{LWIR}-2, \\
11.50-12.50 \mu \mathrm{m}\end{array}$ & - & 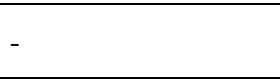 \\
\hline- & 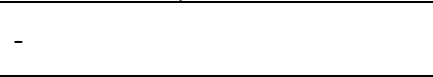 & - & $\begin{array}{l}\text { Bande 9: SWIR 30m } \\
2.360-2.430 \mu \mathrm{m}\end{array}$ \\
\hline- & - & - & $\begin{array}{l}\text { Bande 10: TIR 90m } \\
8.125-8.475 \mu \mathrm{m}\end{array}$ \\
\hline- & - & - & $\begin{array}{l}\text { Bande 11: TIR 90m } \\
8.475-8.825 \mu \mathrm{m}\end{array}$ \\
\hline- & - & - & $\begin{array}{l}\text { Bande 12: TIR 90m } \\
8.925-9.275 \mu \mathrm{m}\end{array}$ \\
\hline
\end{tabular}

Pour les méthodes de traitement des images Aster et Landsat 8 OLI/TIRS qui sont déjà utilisées dans les travaux de recherche précédents, on va les appliquer directement :

Tableau 8: Combinaisons colorées et rapport des bandes d'OLI / TIRS (Landsat 8) et d'ASTER.

\begin{tabular}{|l|l|}
\hline $\begin{array}{l}\text { Combinaisons colorées et rapport des bandes } \\
\text { d'ASTER }\end{array}$ & $\begin{array}{l}\text { Combinaisons colorées et rapport des bandes } \\
\text { d'OLI / TIRS (Landsat 8) }\end{array}$ \\
\hline 468 & $4 / 26 / 75 / 10$ \\
\hline Bandes infrarouges à courte longueur d'onde & \\
\hline
\end{tabular}

\subsection{Création d'un modèle de traitement des images satellitaires}

Le but de cette recherche, n'est pas seulement d'appliquer et évaluer des approches existantes et, aussi, les approches dérivées, mais de créer un modèle scientifique innovant de traitement des images satellitaires pour la prospection minière. Ce modèle est composé par les étapes suivantes : 
a) Prétraitement : Avant de procéder au traitement des images satellitaires, il est indispensable de faire, selon les cas, les corrections géométriques et atmosphériques.

b) Détermination des nombres des combinaisons colorées possibles.

Pour appliquer l'OIF, il est intéressant de déterminer des nombres des combinaisons colorées possibles. Par conséquent, on va utiliser les équations relatives à la mathématique combinatoire présentées dans le tableau récapitulatif ci- après.

Tableau 9: Mathématique combinatoire ( $\mathrm{p}$ et $\mathrm{n}$ appartiennent à l'ensemble des entiers naturels tel que $p \leq n)$.

\begin{tabular}{|c|c|c|}
\hline $\begin{array}{c}\text { Ordonné } \\
\text { (arrangement) }\end{array}$ & $\mathcal{A}_{n}^{p}=\underbrace{n n \ldots n}_{p \text { fois }}=n^{p}$ & $A_{n}^{p}=\frac{n !}{(n-p) !}$ \\
\hline $\begin{array}{c}\text { Non ordonné } \\
\text { (combinaison) }\end{array}$ & $C_{n+p-1}^{p}=\frac{n+p-1 !}{p !(n-1) !}$ & $C_{n}^{p}=\frac{A_{n}^{p}}{p !}=\frac{n !}{p !(n-p) !}$ \\
\hline
\end{tabular}

Dans le calcul, ci-dessous, on prend $\mathrm{p}=3$ puisque chaque combinaison constitue au plus 3 bandes, pour les visualiser en RVB, et $n$ est égale au nombre des bandes.

Tableau 10: Le nombre des combinaisons de bandes.

\begin{tabular}{|c|c|c|c|c|c|c|c|c|c|}
\hline & $\begin{array}{l}\text { Landsat } \\
7 \\
\text { ETM+ }\end{array}$ & $\begin{array}{l}\text { Landsat } \\
8\end{array}$ & \multicolumn{3}{|c|}{ ASTER } & \multicolumn{3}{|c|}{ Sentinel $2 \mathrm{~A}$} & Observations \\
\hline $\begin{array}{l}\text { Nombre des } \\
\text { bandes }\end{array}$ & 8 & 11 & \multicolumn{3}{|l|}{14} & \multicolumn{3}{|l|}{13} & $\begin{array}{l}\text { On va } \\
\text { choisir trois } \\
\text { bandes }\end{array}$ \\
\hline $\begin{array}{l}\text { Arrangements } \\
\text { avec } \\
\text { répétition }\end{array}$ & $8^{3}=512$ & $11^{3}=1331$ & \multicolumn{3}{|c|}{$14^{3}=2744$} & \multicolumn{3}{|c|}{$13^{3}=2197$} & $\begin{array}{l}\text { L'ordre et la } \\
\text { répétition ne } \\
\text { sont pas } \\
\text { demandés }\end{array}$ \\
\hline $\begin{array}{l}\text { Arrangements } \\
\text { sans } \\
\text { répétition }\end{array}$ & 336 & 990 & \multicolumn{3}{|l|}{2184} & \multicolumn{3}{|l|}{1716} & $\begin{array}{l}\text { L'ordre n'est } \\
\text { pas demandé }\end{array}$ \\
\hline $\begin{array}{l}\text { combinaison } \\
\text { avec } \\
\text { répétition }\end{array}$ & 120 & 286 & \multicolumn{3}{|l|}{560} & \multicolumn{3}{|l|}{455} & $\begin{array}{l}\text { La répétition } \\
\text { n'est pas } \\
\text { demandée }\end{array}$ \\
\hline $\begin{array}{l}\text { combinaison } \\
\text { sans } \\
\text { répétition }\end{array}$ & 56 & 165 & \multicolumn{3}{|l|}{364} & \multicolumn{3}{|l|}{286} & $\begin{array}{l}\text { Pour notre } \\
\text { modèle, } \\
\text { combinaison } \\
\text { sans } \\
\text { répétition est } \\
\text { plus adaptée. }\end{array}$ \\
\hline $\begin{array}{l}\text { Nombre des } \\
\text { bandes de }\end{array}$ & $\begin{array}{l}6 \\
(30 \mathrm{~m})\end{array}$ & $8(30 \mathrm{~m})$ & $\begin{array}{l}6 \\
(30 \mathrm{~m})\end{array}$ & $\begin{array}{l}5 \\
(90 \mathrm{~m})\end{array}$ & $\begin{array}{l}3 \\
(15 \mathrm{~m})\end{array}$ & $\begin{array}{l}4 \\
(10 \mathrm{~m})\end{array}$ & $\begin{array}{l}6 \\
(20 \mathrm{~m})\end{array}$ & $\begin{array}{l}3 \\
(60 \mathrm{~m})\end{array}$ & \\
\hline
\end{tabular}




\begin{tabular}{|l|l|l|l|l|l|l|l|l|l|}
\hline $\begin{array}{l}\text { même } \\
\text { résolution }\end{array}$ & & & & & & & & & \\
\hline $\begin{array}{l}\text { combinaison } \\
\text { sans } \\
\text { répétition }\end{array}$ & 20 & 56 & 20 & 10 & & 4 & 20 & 1 & \\
\hline
\end{tabular}

c) Calcul d'Optimum Index Factor :

Pour déterminer la composite colorée la plus importante, on va procéder au calcul de l'OIF comme suit :

Calcul de la matrice de corrélation, on va choisir les bandes de même résolution comme donnée d'entre d'éditeur de modèle spatial. La figure 15 présente le modèle utilisé.

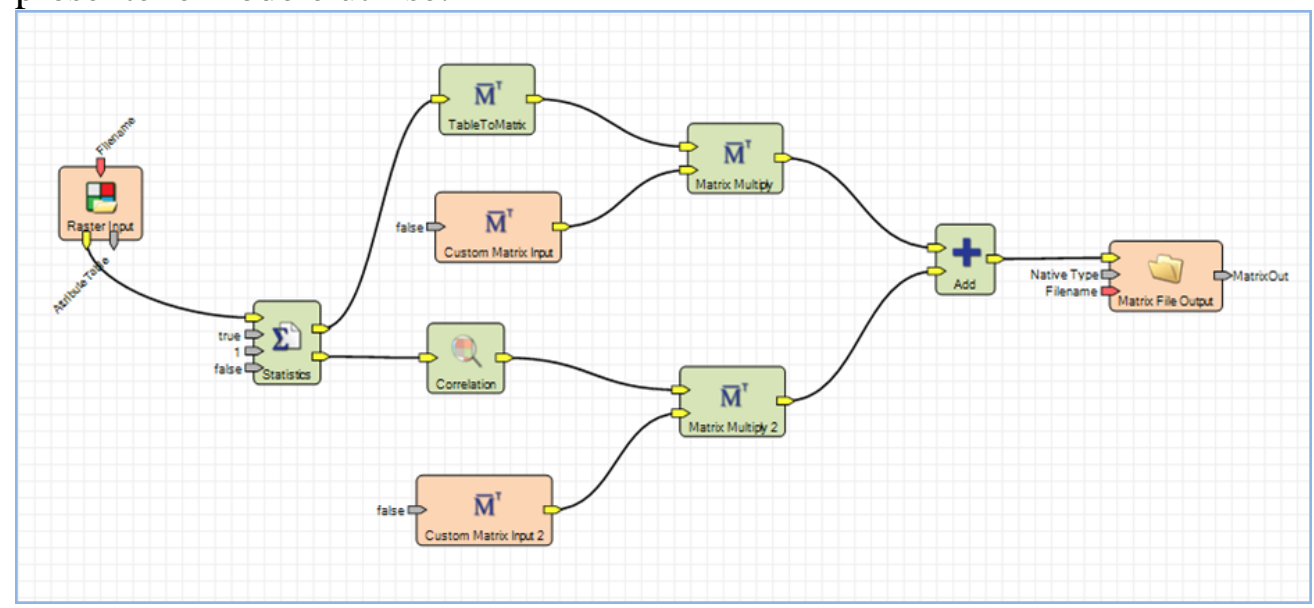

Figure 15 : Modèle spatial sur ERDAS.

On peut illustrer ce modèle par la formule suivante :

(Matrice de covariance * Matrice d'identité) + (Matrice de déviations standards* $[0 \ldots 01])=$ Matrice de corrélation (Tableau 11).

La matrice de covariance et la matrice de standard déviation sont des données de l'image raster. Cette matrice prend la forme suivante :

Tableau 11: Modèle d'une matrice de corrélation.

\begin{tabular}{|c|c|c|c|c|c|c|c|}
\hline Bande & 1 & 2 & 3 & 4 & 5 & 7 & SD \\
\hline 1 & 1 & $0, \ldots$ & $0, \ldots$ & $0, \ldots$ & $0, \ldots$ & $0, \ldots$ & \\
\hline 2 & $0, \ldots$ & 1 & $0, \ldots$ & $0, \ldots$ & $0, \ldots$ & $0, \ldots$ & \\
\hline 3 & $0, \ldots$ & $0, \ldots$ & 1 & $0, \ldots$ & $0, \ldots$ & $0, \ldots$ & \\
\hline 4 & $0, \ldots$ & $0, \ldots$ & $0, \ldots$ & 1 & $0, \ldots$ & $0, \ldots$ & \\
\hline 5 & $0, \ldots$ & $0, \ldots$ & $0, \ldots$ & $0, \ldots$ & 1 & $0, \ldots$ & \\
\hline 7 & $0, \ldots$ & $0, \ldots$ & $0, \ldots$ & $0, \ldots$ & $0, \ldots$ & 1 & \\
\hline
\end{tabular}

On utilise les données de tableaux ci-dessus avec l'équation d'OIF pour calculer les différents termes de ce dernier comme suit : 
Tableau 12: Calcul de l'OIF.

\begin{tabular}{|c|c|c|c|c|}
\hline $\begin{array}{c}\text { Combinaison } \\
\text { des bandes }\end{array}$ & $\begin{array}{l}\text { SOMME } \\
\text { (écart- } \\
\text { type) }\end{array}$ & $\begin{array}{c}\text { SOMME } \\
\text { (corrélation) }\end{array}$ & OIF & rang de l'OIF \\
\hline$\ldots$ & $\ldots$ & $\ldots$ & $\ldots$ & 1 \\
\hline$\ldots$ & $\ldots$ & $\ldots$ & $\ldots$ & 2 \\
\hline$\ldots$ & $\ldots$ & $\ldots$ & $\ldots$. & 3 \\
\hline$\ldots$ & $\ldots$ & $\ldots$ & $\ldots$ & 4 \\
\hline$\ldots$ & $\ldots$ & $\ldots$ & $\ldots$. & 5 \\
\hline$\ldots$ & $\ldots$ & $\ldots$ & $\ldots$ & $\ldots$ \\
\hline
\end{tabular}

D'après ce calcul d'OIF, on peut bien déterminer les composantes colorées les plus importantes qui ne représentent pas des redondances selon l'ordre croissant de leur OIF.

d) Les rapports des bandes (Rations)

D'après la littérature, il existe plusieurs rapports des bandes déjà validés par leurs bons résultats.

En général, on peut calculer le nombre des possibilités des rapports des bandes, on prend $\mathbf{p}=2$ puisque chaque rapport est constitué de deux bandes, et $\mathbf{n}$ est égale au nombre des bandes.

Tableau 13: Le nombre des rapports des bandes possibles.

\begin{tabular}{|c|c|c|c|c|c|c|c|c|}
\hline & Landsat 7 & Landsat 8 & \multicolumn{3}{|c|}{ Sentinel 2 A } & \multicolumn{3}{|c|}{ ASTER } \\
\hline $\begin{array}{c}\text { Nombre des } \\
\text { bandes }\end{array}$ & 6 & 8 & 4 & 6 & 3 & 3 & 6 & 5 \\
\hline Résolution & $30 \mathrm{~m}$ & $30 \mathrm{~m}$ & $10 \mathrm{~m}$ & $20 \mathrm{~m}$ & $60 \mathrm{~m}$ & $\begin{array}{l}15 \\
\mathrm{~m}\end{array}$ & $30 \mathrm{~m}$ & $90 \mathrm{~m}$ \\
\hline $\begin{array}{l}\text { Combinaison } \\
\text { sans } \\
\text { répétition }\end{array}$ & 15 & 28 & 6 & 15 & 3 & 3 & 15 & 10 \\
\hline $\begin{array}{l}\text { Rations } \\
\text { possibles }\end{array}$ & $\begin{array}{lll}1 / 2 & 1 / 3 & 1 / 4 \\
1 / 5 & 1 / 6 & 2 / 3 \\
2 / 4 & 2 / 5 & 2 / 6 \\
3 / 4 & 3 / 5 & 3 / 6 \\
4 / 5 & 4 / 6 & 5 / 6\end{array}$ & $\begin{array}{llll}1 / 2 & 1 / 3 & 1 / 4 & 1 / 5 \\
1 / 6 & 1 / 7 & 1 / 9 & 2 / 3 \\
2 / 4 & 2 / 5 & 2 / 6 & 2 / 7 \\
2 / 9 & 3 / 4 & 3 / 5 & 3 / 6 \\
3 / 7 & 3 / 9 & 4 / 5 & 4 / 6 \\
4 / 7 & 4 / 9 & 5 / 6 & 5 / 7 \\
5 / 9 & 6 / 7 & 6 / 9 & 7 / 9\end{array}$ & $\begin{array}{l}2 / 3 \\
2 / 4 \\
2 / 8 \\
3 / 4 \\
3 / 8 \\
4 / 8\end{array}$ & $\begin{array}{c}5 / 65 / 7 \\
5 / 8 \mathrm{~A} 5 / 11 \\
5 / 126 / 7 \\
6 / 8 \mathrm{~A} 6 / 11 \\
6 / 127 / 8 \mathrm{~A} \\
7 / 117 / 12 \\
8 \mathrm{~A} / 11 \\
8 \mathrm{~A} / 12 \\
11 / 12\end{array}$ & $\begin{array}{c}1 / 9 \\
1 / 10 \\
9 / 10\end{array}$ & $\begin{array}{l}1 / 2 \\
1 / 3 \\
2 / 3\end{array}$ & $\begin{array}{c}4 / 54 / 6 \\
4 / 74 / 8 \\
4 / 95 / 6 \\
5 / 75 / 8 \\
5 / 96 / 7 \\
6 / 86 / 9 \\
7 / 87 / 9 \\
8 / 9\end{array}$ & $\begin{array}{l}10 / 11 \\
10 / 12 \\
10 / 13 \\
10 / 14 \\
11 / 12 \\
11 / 13 \\
11 / 14 \\
12 / 13 \\
12 / 14 \\
13 / 14\end{array}$ \\
\hline
\end{tabular}

Les rations, mentionnées dans le tableau 13, sont des nouvelles bandes qui peuvent constituer la base pour l'application de l'OIF pour déterminer les combinaisons les plus importantes.

Et dans ce cas, le nombre des composantes colorées possibles est illustré dans le tableau ci-après. 
Tableau 14: Le nombre des composantes colorées possibles des ratios.

\begin{tabular}{|c|c|c|c|c|c|c|c|c|}
\hline & $\begin{array}{c}\text { Landsat } \\
7 \text { ETM+ }\end{array}$ & $\begin{array}{c}\text { Landsat } \\
8\end{array}$ & \multicolumn{3}{|c|}{ ASTER } & \multicolumn{3}{c|}{ Sentinel 2 A } \\
\hline $\begin{array}{c}\text { Nombre des } \\
\text { bandes de même } \\
\text { résolution }\end{array}$ & $\begin{array}{c}6 \\
(30 \mathrm{~m})\end{array}$ & $\begin{array}{c}8 \\
(30 \mathrm{~m})\end{array}$ & $\begin{array}{c}6 \\
(30 \mathrm{~m})\end{array}$ & $\begin{array}{c}5 \\
(90 \mathrm{~m})\end{array}$ & $\begin{array}{c}3 \\
(15 \mathrm{~m})\end{array}$ & $\begin{array}{c}4 \\
(10 \mathrm{~m})\end{array}$ & $\begin{array}{c}6 \\
(20 \mathrm{~m})\end{array}$ & $\begin{array}{c}3 \\
(60 \mathrm{~m})\end{array}$ \\
\hline $\begin{array}{c}\text { Nombre des } \\
\text { rations }\end{array}$ & 15 & 28 & 15 & 10 & 3 & 6 & 15 & 3 \\
\hline $\begin{array}{c}\text { Nombre des } \\
\text { composantes } \\
\text { colorées possibles }\end{array}$ & 455 & 3276 & 445 & 120 & 1 & 20 & 445 & 1 \\
\hline
\end{tabular}

e) Analyse en composante principale

L'Analyse en Composantes principales, ACP, fait partie du groupe des méthodes descriptives multidimensionnelles appelées méthodes factorielles. L'ACP propose, à partir d'un tableau rectangulaire de données comportant les valeurs de $\mathrm{p}$ variables quantitatives pour $\mathrm{n}$ unités, des représentations géométriques de ces unités et de ces variables. L'ACP servira à mieux connaitre les données sur lesquelles on travaille, et aidera à formuler des hypothèses de résolution qu'il faudra étudier et faciliter les représentations fournies pour illustrer certains résultats (Duby \& Robin).

f) Amélioration radiométrique et spatiale par fusion

L'amélioration radiométrique est une transformation virtuelle des données pour utiliser toute la gamme d'intensité disponible afin de visualiser les données à l'écran. Cette amélioration ne modifie donc pas les données sources (la valeur des pixels). Elle change les valeurs initiales de façon à utiliser toutes les valeurs possibles, ce qui permet d'augmenter le contraste entre les cibles et leur environnement. Pour mieux comprendre le fonctionnement de cette amélioration, il faut d'abord comprendre le concept de l'histogramme d'une image qu'est une représentation graphique des valeurs numériques d'intensité qui constituent une image. Ces valeurs (de 0 à 255 pour des données à 8 bits) apparaissent le long de l'axe des x du graphique. La fréquence d'occurrence de chacune de ces valeurs est présentée par le long de l'axe des y (El Morjani 2015).

Les satellites d'observation de la Terre fournissent des données multispectrales et panchromatiques ayant différentes résolutions spatiales, spectrales, temporelles, et radiométriques. La fusion d'une image panchromatique ayant une haute résolution spatiale, mais une basse résolution spectrale avec une image multispectrale ayant une basse résolution spatiale mais une haute résolution spectrale est très utile dans de nombreuses applications de télédétection nécessitant à la fois de hautes résolutions spatiales et spectrales. Dans l'image résultante les structures et les objets sont bien rehaussés et la classification de ces objets devient plus précise. Ces techniques de traitement d'image sont connues sous le nom de pansharpening 
ou techniques de fusion de résolutions. Nous pouvons, à titre d'exemple fusionner une image SPOT multispectrale $(20 \mathrm{~m})$ avec une autre image SPOT panchromatique $(10 \mathrm{~m})$, ou une image Landsat ETM+ $(30 \mathrm{~m}$ pour les bandes VIR) avec son canal panchromatique (15m) (El Mezouar 2012).

g) Analyse structurale et cartes de linéaments

Les filtres directionnels peuvent être, ensuite, appliqués aux différentes bandes spectrales et aux néo-canaux ( $\mathrm{CP}$, et bandes ratios) en vue d'obtenir les cartes de linéaments. Le filtrage d'une image permet de lui appliquer une fonction mathématique qui modifie les valeurs du gris de tout ou une partie des pixels. Dans le cas d'une fonction linéaire, le filtrage est dit linéaire, cependant, dans le cas de prise en compte des valeurs du gris au voisinage de chaque pixel transformé, elle est dite fonction de convolution. Les méthodes utilisées, généralement, sont de type convolution :

- Le filtre Sobel : utilisé pour détecter les contours d'objets dans une image, en faisant deux balayages l'un horizontal et l'autre vertical. Il donne souvent de bons résultats pour la détection des linéaments.

- Les filtres directionnels : ils améliorent la perception des linéaments, correspondants à des discontinuités lithologiques ou structurales, en provoquant un effet optique d'ombre porté sur l'image.

h) Classification des images satellitaires (non supervisée et supervisée) La classification d'image est une interprétation assistée par ordinateur d'images satellitaires, et se définit comme l'opération qui regroupe au mieux de leur ressemblance spectrale les divers objets de la cible. Autrement dit c'est une méthode d'analyse des données qui permet de ranger des pixels de l'image dans des classes homogènes. Il existe deux approches pour la classification d'images, une est dite non supervisée, qui est utilisée dans le cas où l'information qu'on dispose est faible sur la zone étudiée (le logiciel définit lui-même les classes); l'autre est dite supervisée et nécessite une bonne connaissance de la zone d'étude (l'utilisateur définit lui-même les classes à produire (nombre, caractéristique spectrale,...) (EL MORJANI 2015).

\section{Conclusions}

À travers cet état de connaissances, on a essayé de rassembler plusieurs études antérieures afin de créer un modèle de traitement des images satellitaires multibandes ; on peut résumer ce modèle dans le schéma illustré par la figure 16 : 


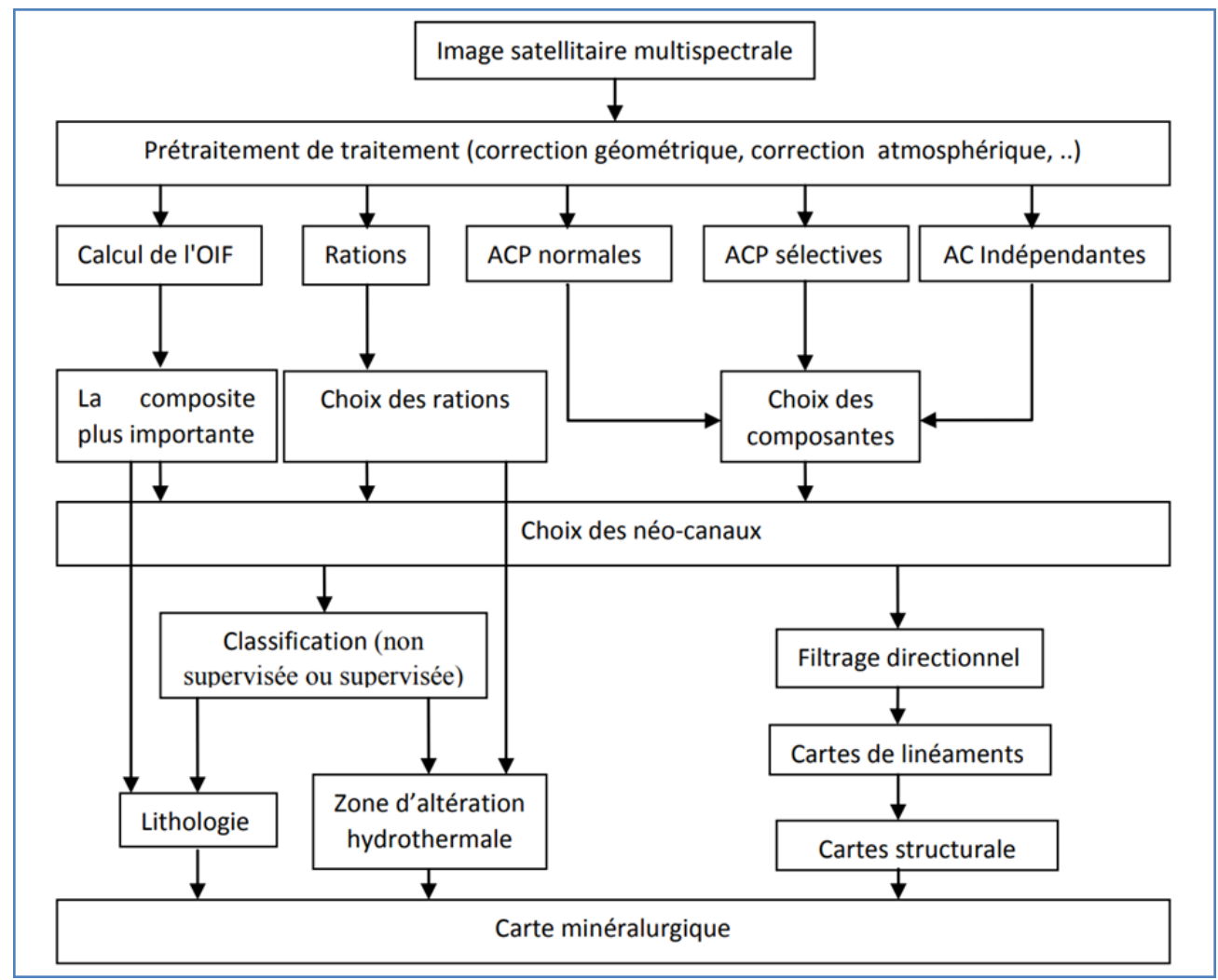

Figure 16 : Schéma récapitulatif du modèle de traitement.

Ce modèle nous permet d'identifier les sites probablement miniers par l'analyse des données lithologiques et structurales résultantes de la méthode de traitement d'image, surtout, qui sont liées à la zone d'altération hydrothermale. Toutefois, ces résultats sont confrontés pendant le processus d'étude à des données du terrain existant afin de les valider.

Il est à signaler que ce modèle de traitement des images satellitaires ne substitue pas les méthodes conventionnelles de la recherche minière, cependant il reste d'une grande utilité en prospection pour réduire à la fois le coût et le temps surtout dans les sites à accès difficile.

Ces résultats positifs de l'imagerie multispectrale, dans les travaux de recherche précédents, permettent d'identifier les zones favorables à contenir la minéralisation. Et puisque, en général, les failles et les différentes formes de minéralisation ne dépassent pas, actuellement, quelque mètre, d'où la nécessité de l'usage d'images spatiales ou aériennes à grande résolution. Les travaux futurs seront bien développés aussi pour l'imagerie hyperspectrale et l'imagerie Radar pour évaluer leur potentiel notamment si on les associe avec d'autres données de la géophysique et la géochimie. 


\section{References:}

1. Achek, H., \& Aidouni, N.-e. (2014). Essai de cartographie géologique par la télédétection optique de la région Hank (Sud-Ouest Algérien).

2. Adiri, Z., El-Harti, A., Jellouli, A., Maacha, L., \& Bachaoui, E.-M. (2016). Lithological mapping using Landsat 8 and ASTER multispectral data in the Bas Drâa inlier, Moroccan Anti Atlas. Journal of Applied Remote Sensing .

3. Al Hakim, A. Y., \& Sulistijo, B. (2013 ). Integrated Exploration Method to Determine Cu Prospect In Seweden District, Blitar, East Java. Procedia Earth and Planetary Science , $64-69$.

4. Amri, K., Mahdjoub, Y., \& Guergour, L. (Novembre 2009). Apport des images Landsat 7 ETM+ pour la discrimination lithologique et l'2tude structurale de la région d'Afara Héouine, Tahifet, Hoggar Central. Journées d'Animation Scientifique (JAS09) de l'AUF Alger .

5. Chavez, \& al. (1982). Optimum Index Factor.

6. Dabo, M., \& Aïfa, T. (2013). architecture tectonique des formations paleoproterozö̈ques de la dalema (senegal oriental): apport des lineaments d'images landsat-7 etm $+/$ srtm et aeromagnetique. Journal des Sciences, 32-45.

7. Duby, C., \& Robin, S. Analyse en Composantes Principales. AgroParisTech.

8. El Harti, A., Bannari, A., Bachaoui, E. M., Aarab, E. M., Girouard, G., \& El Ghmari, A. (2004). Synergie entre la télédétection multispectrale et les données de terrain pour la conception d'un nouveau modèle géodynamique d'ouverture du bassin paléozoïque des Jebilet centrales (Maroc).C. R. Geoscience336 , 1311-1320.

9. El Harti, A., Aarab, E. M., Bachaoui, M., \& El Ghmari, A. (2006). Réponse au commentaire de A. Essaifi et al., sur la note Synergie entre la télédétection multispectrale et les données de terrain pour la conception d'un nouveau modèle géodynamique d'ouverture du bassin paléozoïque des Jebilet centrales (Maroc). C. R. Geoscience 338 , 389-391.

10. El Mezouar, M. C. (2012). Fusion d'images en télédétection satellitaire (Thèse). INSA de Rennes; Université Djillali Liabes de Sidi Bel Abbés.

11. El Morjani, Z. E. A. (2015). initiation a la teledetection spatiale : travaux pratiques. FPT-UIZ.

12. Essaifi, A., Lagarde, J.-L., \& Capdevila, R. (2006). Commentaire à la note intitulée Synergie entre la télédétection multispectrale et les données de terrain pour la conception d'un nouveau modèle géodynamique d'ouverture du bassin paléozoïque des Jebilet centrales (Maroc), de A. El Harti et al. C. R. Geoscience 338 , 386-388. 
13. Hamzaoui A.: Télédétection multispectrale et hyperspectrale appliquée à l'exploration minière. Journées nationales de l'industrie minérale. 23-25 Novembre 2005, Marrakech.

14. Hakdaou, M., Emran, A., \& Rahim, A. (2005). fusion d'images optique et radar: application a la cartographie geologique dans la region du haut atlas central marocain. Workshop International Télédétection et Systèmes d'Information Géographique "Information Spatiale et Développement Durable"., (p. 35). RABAT.

15. Harris, J., \& al. (s.d.). Hyperspectral Remote Sensing Hyperspectral Remote Sensing for Geological Mapping and for Geological Mapping and Mineral Exploration Mineral Exploration. A Review of Activities at NRCAN .

16. IHMC Public Cmaps. (s.d.). Consulté le 02 28, 2017, sur http://skat.ihmc.us/rid=1HRMCV1DD-TBH7CY-

Z4K/T\%C3\%A91\%C3\%A9d\%C3\%A9tection.cmap

17. Imessaoudene, N. (2012). Utilisation de la télédétection pour la cartographie géologique du Massif des Eglab et de sa bordure sédimentaire (Sud-Ouest algérien). MEMOIRE DE FIN D'ETUDE, UNIVERSITE FERHAT ABBAS - SETIF, INSTITUT D'ARCHITECTURE \& DES SCIENCES DE LA TERRE.

18. Mike, A. (2005, 08 02). Les données Aster.

19. NASA. (s.d.). ASTER Instrument Characteristics. Consulté le 12 22, 2016, sur ASTER web: http://asterweb.jpl.nasa.gov/characteristics.asp

20. NASA. (s.d.). Geology. Consulté le 01 01, 2017, sur ASTER WEB: http://asterweb.jpl.nasa.gov/content/03_data/05_Application_Exampl es/geology/default.HTM

21. NASA. (s.d.). Landsat. Consulté le 12 21, 2016, sur Landsat science: http://landsat.gsfc.nasa.gov/landsat-8/landsat-8-overview/

22. NASA, \& USGS. (2012, Decembre). Landsat Data Continuity Mission: Continuously Observing Your World.

23. NASA, METI, AIST, Systems, J. S., \& Team, U. A. (s.d.). Consulté le 12 22, 2016, sur ASTER: http://asterweb.jpl.nasa.gov/gallerydetail.asp?name $=$ atlas

24. ONHYM (s.d.). Consulté le 01 15, 2017, sur http://www.onhym.com/: http://www.onhym.com/methodes-d-exploration/teledetection.html

25. Ouattara, G., Koffi, G. B., \& Yao, A. K. (2012). Contribution des images satellitales Landsat 7 ETM + à la cartographie lithostructurale du Centre-Est de la Cote d'Ivoire (Afrique de l'Ouest).International Journal of Innovation and Applied Studies , 61-75.

26. Pour, A., \& Hashim, M. (2014). Hydrothermal alteration mapping using Landsat-8 data, Sar Cheshmeh copper mining district, SE Iran. Journal of Taibah University for Science . 
27. Qaid, A. M., \& Basavarajappa, H. (2008). Application of Optimum Index Factor Technique to Landsat-7 Data for Geological Mapping of North East of Hajjah, Yemen. American-Eurasian Journal of Scientific Research, 84-91.

28. Rashmi, S., Swapna, A., Venkat, S., \& Ravikiran, S. (2014). Spectral Angle Mapper Algorithm for Remote Sensing Image Classification. IJISET - International Journal of Innovative Science, Engineering \& Technology , 1 (4).

29. Shaddad, I. A. (2005). Application of remote sensing techniques for regional geological survey in some selected areas, sudan.Workshop International Télédétection et Systèmes d'Information Géographique "Information Spatiale et Développement Durable", (pp. 35-36). RABAT.

30. Smith, R. E., Green, A. A., Robinson, G., \& Honey, F. R. (1978). use of landsat -1 imagery in exploration for keweenawan - type copper deposits. REMOTE SENSING OF ENVIRONMENT , 129-144.

31. Spatial-analyst.net. (s.d.). Optimum Index Factor. Consulté le 01 15, 2017, sur http://spatial-analyst.net/: http://spatialanalyst.net/ILWIS/htm/ilwisapp/optimum_index_factor_functionality _algorithm.htm

32. Taibi, D. (2009).

33. USGS, N. (2010, 11 19). Landsat : Data Continuity Mission.

34. Zhang, Y.-J., \& Yao, F.-J. (2015). Interpreting the Shortwave Infrared \& Thermal Infrared Regions of Remote Sensed Electromagnetic Spectrum with Application for Mineral-Deposits Exploration. Journal of Applied Mathematics and Physics, 254-261. 